\title{
Catálogo de Apoidea da Região Neotropical (Hymenoptera, Colletidae). I. Paracolletini ${ }^{1}$
}

\author{
Jesus S. Moure ${ }^{2}$ \\ Vinalto Graf ${ }^{2}$ \\ Danúncia Urban ${ }^{2}$
}

\begin{abstract}
Catalog of the Apoidea of the Neotropical Region (Hymenoptera, Colletidae). I. Paracolletini. The species of the tribe Paracolletini are presented as the first part of a commented catalog of Colletidae bees, from the Neotropical Region. The geographic distribution, flowers, biological behaviour, taxonomy, type locality and depositary museums are mentioned, bibliographic information are given for each species and a new genus, Actenosigynes, is proposed to Leioproctus (L.) fulvoniger Michener, 1989.

KEY WORDS. Hymenoptera, Apoidea, Colletidae, Paracolletini, Neotropical, catalogue
\end{abstract}

Este trabalho mantém a estrutura apresentada por MOURE \& HURD (1987) para os Halictidae, com tópicos de revisão, taxonomia, biologia e morfologia.

MICHENER (1944) considerou os Paracolletini como o grupo mais primitivo das abelhas, citou a sua distribuição geográfica na América do Sul, África e Austrália e listou os gêneros incluidos.

MOURE (1945) propôs Lonchopriinae para os gêneros: Lonchopria Vachal, 1905, Nomiocolletes Brèthes, 1909, Halictanthrena Ducke, 1907, Biglossa Friese, 1906, Bicolletes Friese, 1908, Perditomorpha Ashmead, 1899 e Brachyglossula Hedicke, 1922, considerados no trabalho de MiCHENER (1944) como Paracolletini; e para Eulonchopria Brèthes, 1909, formou mais uma sub-família: Eulonchopriinae.

MiCHENER (1965) ao estudar as abelhas australianas e da região sul do Pacífico estendeu a distribuição de Leioproctus Smith, 1853 à América do Sul, com grande número de sub-gêneros e espécies.

MICHENER (1989), apresentou uma revisão dos gêneros de Paracolletini e Colletini Americanos e comentou que dada a natureza não resolvida da análise cladística dos Paracolletini Americanos e Australianos, ainda não seria possível uma análise biogeográfica conclusiva; e descreveu 18 sub-gêneros de Leioproctus para a região Neotropical. A interpretação de Michener (1989) para os Paracolletini neotropicais, como pertencentes ao gênero Leioproctus, com L. imitatus Smith, 1853 como espécie-tipo, da Nova Zelândia, não foi seguida neste trabalho; e são

1) Contribuição número 1046 do Departamento de Zoologia, Universidade Federal do Paraná.

2) Departamento de Zoologia, Universidade Federal do Paraná. Caixa Postal 19020, 81531-990 Curitiba, Paraná, Brasil. Bolsista do CNPq. 
considerados com o status de gênero, os seguintes sub-gêneros propostos por MiCHENER (1989): Chilicolletes, Glossopasiphae, Kylopasiphae, Pygopasiphae, Reedapis e Torocolletes. São revalidados como gêneros: Nomiocolletes Brèthes, 1909; Hoplocolletes Michener, 1965; Edwinyana Moure, 1954. Em trabalhos anteriores foram colocados na categoria genérica: Sarocolletes Michener, 1989 por MoURE \& URBAN (1995); Cephalocolletes Michener, 1989 por URBAN (1995) e, Protodiscelis Brèthes, 1909 e Tetraglossula Ogloblin, 1948, por Melo (1996), todos sub-gêneros para MiCHENER (1989).

Com relação a Lonchopria Vachal, 1905, MichENER (1989) juntou gêneros, considerando-os como sub-gêneros, comentando textualmente (p. 681): "Lonchopria s.str. is quite different from other subgenera, and might have been regarded as a genus separate from Biglossa and Porterapis except for the intermediacy of Ctenosibyne"; sobre Biglossa (p. 676), comentou que a maioria das espécies poderia ser colocada no sub-gênero Biglossidia Moure, 1948 e que então apenas uma espécie permaneceria no sub-gênero Biglossa; as figuras que ilustram o trabalho de Michener destacam as diferenças entre os citados sub-gêneros. Desta forma são considerados gêneros válidos, distintos de Lonchopria: Ctenosibyne Moure, 1956; Porterapis Michener 1989 e Lonchoprella Michener 1989. Também um novo status é proposto para Ethalonchopria Michener, 1989, o qual foi previamente considerado como sub-gênero de Eulonchopria.

MiCHENER (1997) publicou uma lista alfabética dos nomes genéricos e sub-genéricos das abelhas, com a indicação das espécies tipo e comentários taxonômicos, onde são citados os Paracolletini.

Neste catálogo as informações bibliográficas são dadas abreviadamente quando repetidas no gênero ou na espécie, as mais frequentes estão listadas nas Referências Bibliográficas; as citações, listas de espécies, e redescrições não pertinentes não são mencionadas. As abreviações latinas são as comumente encontradas em trabalhos de taxonomia.

Os acrônimos dos museus citados no texto são relacionados a seguir: (AMNH) American Museum of Natural History, Nova York, Estados unidos da América; (BMNH) The Natural History Museum, Londres, Inglaterra; (CAS) California Academy of Sciences, San Francisco, Estados Unidos da América; (CTV) Coleção Toro, Valparaíso, Chile; (DMHN) Divisão de Museu de História Natural, Capão da Imbuia, Curitiba, Brasil; (DZUP) Departamento de Zoologia da Universidade Federal do Paraná, Coleção Pe. J.S. Moure, Curitiba, Brasil; (IML) Instituto Miguel Lillo, Universidad Nacional de Tucumán, San Miguel de Tucumán, Argentina (inclui a Coleção Arnau); (IZUC) Instituto de Zoología, Universidad de Concepción, Concepción, Chile (inclui a Coleção Wagenknecht); (MBR) Museo Argentino de Ciencias Naturales "Bernardino Rivadavia", Buenos Aires, Argentina; (MEUV) Museu Entomológico da Universidade Federal de Viçosa, Minas Gerais, Brasil; (MHNS) Museo Nacional de Historia Natural, Santiago, Chile; (MHPN) Museu de Historia Natural, Colégio San Pedro Nolasco, Santiago, Chile (inclui a Coleção Ruiz); (MIZT) Museo ed Istituto di Zoologia Sistematica, Universitá di Torino, Turim, Itália; (MJP) Museo de Historia Natural, Universidad Nacional 
Mayor de San Marcos, Lima, Peru; (MLP) Facultad de Ciencias Naturales y Museo, Universidad Nacional de La Plata, La Plata, Argentina (inclui as Coleções Ogloblin e Joergensen); (MNHP) Muséum National d'Histoire Naturelle, Paris, França (inclui a Coleção Vachal); (MNHU) Museum für Naturkunde der Humboldt-Universität zu Berlin, Alemanha (inclui as Coleções Herbst, Friese e Strand); (MNRJ) Museu Nacional, Rio de Janeiro, Brasil (inclui a Coleção Campos Seabra); (MPEG) Museu Paraense Emílio Goeldi, Belém, Brasil; (MZSP) Museu de Zoologia, Universidade de São Paulo, Brasil (ex Departamento de Zoologia da Secretaria de Agricultura); (SEMK) Snow Entomological Museum, University of Kansas, Lawrence, Estados Unidos da América; (UAT) University of Arizona, Tucson, Estados Unidos da América; (UCRS) Laboratório de Pesquisas Biológicas da Pontifícia Universidade Católica do Rio Grande do Sul, Brasil; (UCVC) Laboratório de Zoología de la Universidad Católica de Valparaíso, Chile; (USNM) National Museum of Natural History, Washington, Estados Unidos da América.

Quanto à Coleção Schrottky, não é conhecido o paradeiro da parte depositada no Paraguai, os demais espécimens estão depositados no MBR, MZSP e Instituto Oswaldo Cruz, Rio de Janeiro, Brasil.

\section{Paracolletini}

\section{Actenosigynes Moure; Graf \& Urban gen.n.}

Espécie-tipo: Leioproctus fulvoniger Michener.

Leioproctus (Leioproctus) (partim) Michener, 1989. Univ. Kansas Sci. Bull. 53 (11): 641- 643.

O gênero Actenosigynes está sendo proposto para Leioproctus (Leioproctus) fulvoniger Michener, 1989, única espécie sulamericana atribuída por Michener a Leioproctus s.str.

Preto, tergos não metálicos e sem faixas amarelas. Labro, glossa e palpos curtos, a glossa emarginada em arco; área malar estreita, menor que a metade do diâmetro do flagelo; clípeo aproximadamente duas vezes tão largo como longo; distância clipeocelar menos de duas vezes o comprimento do clípeo; supraclipeal brilhante e pontuada, com elevação angulosa entre os alvéolos; carena frontal bem marcada; borda occipital arredondada; escapo tão longo como a distância alveolocelar; flagelômero basal tão longo como largo, o segundo curto, metade da sua largura. Pronoto e parte anterior do mesepisterno não arestados; mesoscuto e escutelo brilhantes e com pontuação densa, intervalos iguais ou menores que o diâmetro dos pontos; base do propódeo denso-reticulada e transversalmente estriada, um pouco mais curta que o metanoto; triângulo propodeal denso-reticulado, quase fosco, bastante inclinado, fortemente limitado pelos sulcos espiraculares, estes formando aréolas subquadradas na parte superior; asas anteriores com três células sub-marginais, a segunda pequena, tão curta no bordo posterior como a metade do bordo posterior da terceira sub-marginal, subquadrada, recebendo o primeiro médio-cubital pouco depois do meio; asas posteriores com nove hâmulos, lobo jugal dois terços do lobo anal; placa basitibial pilosa com as bordas bem marcadas. 
Machos com labro muito estreito, sem área mate na metade distal, deprimido no meio ao longo do comprimento; antenas sobrepassando as tégulas; mandíbulas fracamente bidentadas o dente interno superior fraco; tíbias posteriores com cerdas ramificadas e lisas como na fêmea; sem placa pigidial, somente com área mediana lisa e irregular; sétimo esterno com os apódemas maiores que as placas laterais, estas bilobadas, o lobo distal um pouco mais longo e mais estreito que o basal.

Fêmeas com labro quatro vezes tão largo como longo, com depressão discal e área mate na metade basal; placa pigidial fortemente pontuada, mais lisa para a borda; esporão interno das tíbias posteriores com dentículos curtos e numerosos, terminando em ponta reta e aguda; escopa tibial moderadamente densa sem ocultar o tegumento, no lado externo predominam cerdas com ramificações finas em um dos lados, e com cerdas lisas na face interna; basitarso posterior com predominância de cerdas lisas na face externa, mais longas que na face interna. Parte distal dos esternos com pêlos inclinados para trás, numa área pouco maior que a metade exposta dos esternos.

A única espécie incluida no gênero, Actenosigynes fulvoniger (Michener, 1989), é tipicamente uma espécie do sul da Mata Atlântica. Foi descrita de Corupá, Santa Catarina e são acrescentadas as novas ocorrências: serra da Graciosa [Morretes], Paraná e Osório no Rio Grande do Sul.

Comentários. Actenosigynes tem em comum com Halictanthrena, Homlbergeria e Chilicolletes a segunda célula sub-marginal muito curta, aproximadamente a metade do comprimento da terceira no bordo posterior; entretanto Halictanthrena tem placas laterais inteiras no sétimo esterno do macho; Holmbergeria tem as placas laterais do sétimo esterno bífidas e muito longas; e Chilicolletes tem as placas laterais do sétimo esterno bífidas porém curtas como em Actenosigynes. O macho de Chilicolletes distingue-se pelas tíbias posteriores com cerdas longas densamente micro-ramificadas e as fêmeas pelo esporão interno pectinado.

Etimologia. Do grego: "actenos" = não pectinado + "sigynes" = esporão. (Masculino).

Taxonomia. Michener, 1989: 641-643 (redescrição e nota comparativa; inclui $L$. (L.) fulvoniger).

fulvoniger Michener, comb.n. BRASIL, Santa Catarina: Corupá, Seara (Nova Teutônia) (localidade tipo); Paraná: Morretes; Rio Grande do Sul: Osório.

Leioproctus (Leioproctus) fulvoniger Michener, 1989. Univ. Kansas Sci. Bull. 53 (11): 643, 691-692; figs 5 g-l, 19 c. Holótipo macho e alótipo no SEMK.

Taxonomia. Michener, 1989: 691 (única espécie deste sub-gênero nas Américas).

Biologia. Wittmann \& Schlindwein, 1995. Biociências, Porto Alegre, 3 (2): 19-34 (observaram machos dormindo nas flores, machos e fêmeas polinizando flores de Cajophora eichleri). 


\section{Aeganopria Moure}

Aeganopria Moure, 1949. Rev. Ent., Rio de Janeiro, 20 (1/3): 442. - Michener, 1997. Sci. Papers, Nat.

Hist. Mus., Univ. Kansas 1: 4.

Espécie-tipo: Lonchopria nivosa Vachal, 1909. Designação original.

Leioproctus (Aeganopria); Michener, 1964. Systematic Zool. 13 (4): 187. - Michener, 1965. Bull.

Amer. Mus. Nat. Hist. 130: 41.

Lonchopria (Biglossa) (partim); Michener, 1989. Univ. Kansas Sci. Bull. 53 (11): 673-678.

Taxonomia. Moure, 1949. Rev. Ent., Rio de Janeiro, 20 (1/3): 442-444 (considerou próximo ao gênero Biglossa, citando caracteres diferenciais). - Moure, 1956. Dusenia, Curitiba, 7 (6): 311 (sugeriu que Aeganopria, Lonchopria s.str., Ctenosibyne e Biglossa seriam reunidos sob a denominação genérica de Lonchopria). - Michener, 1989: 673 (Aeganopria como sinônimo do sub-gênero Biglossa); 678 (considerou que poderia ser sub-gênero e grupo-irmão de Biglossa).

nivosa (Vachal). ARgENTINA, Tucumán (localidade tipo), Tacanas, Tapia; Salta, Tabacal.

Lonchopria nivosa Vachal, 1909. Rev. d'Ent., Caen, 28: 36. Holótipo fêmea no MNHP.

Aeganopria nivosa; Moure, 1949. Rev. Ent., Rio de Janeiro, 20 (1/3): 444-445.

Lonchopria (Biglossa) nivosa; Michener, 1989. Univ. Kansas Sci. Bull. 53 (11): 673- 678 .

Taxonomia. Cockerell, 1917. Ann. Mag. Nat. Hist. (8) 20: 440 (comparou com L. rufipennis). - Michener, 1989: 677 (fig. 27, i-o). - Moure, 1949: 444 (descreveu o macho).

\section{Belopria Moure}

Belopria Moure, 1956. Dusenia, Curitiba, 7 (6): 305. - Michener, 1997. Sci. Papers, Nat. Hist. Mus., Univ. Kansas 1: 10.

Espécie-tipo: Belopria zonata Moure, 1956. Designação original.

Leioproctus (Belopria); Michener, 1964. Systematic Zool. 13 (4): 187. - Michener, 1965. Bull. Amer.

Mus. Nat. Hist. 130: 41.

Leioproctus (Perditomorpha).(partim); Michener, 1989. Univ. Kansas Sci. Bull. 53 (11): 635

Taxonomia. Moure, 1964, Bol. Univ. Paraná, Zoo., 2 (4): 55 (considerou este gênero próximo de Niltonia, Hexantheda, Tetraglossula, Perditomorpha e Bicolletes). - Michener, 1989: 635-639 (comentou que Belopria poderia ser reconhecido como sub-gênero mas fracamente diferenciado dos outros e assim preferiu colocar suas espécies em Perditomorpha).

nitidior Moure. BRASIL, Rio de Janeiro, Itatiaia (localidade tipo), 1.300-2. 200m; Paraná, Lapa.

Belopria nitidior Moure, 1956. Dusenia, Curitiba, 7 (6): 309-311. Holótipo fêmea e alótipo no DZUP.

Leioproctus (Perditomorpha) nitidior; Michener, 1989. Univ. Kansas Sci.

Bull. 53 (11): 635, 636, 638, 639.

Taxonomia. Michener, 1989: 635-639 (nota comparativa). 
Biologia. Barbola \& Laroca, 1995. Acta Biol. Par., Curitiba, 22 (1993) (1-4): 95 (diversidade e abundância relativa, na Lapa, PR).

zonata Moure. BrasiL, Paraná: Curitiba, 900m (localidade tipo); S. José dos Pinhais, $880 \mathrm{~m}$.

Belopria zonata Moure, 1956. Dusenia, Curitiba, 7 (6): 307-309, figs 1-3. Holótipo fêmea, alótipo e parátipos no DZUP; parátipos no DMHN, MZSP, MNRJ e SEMK.

Leioproctus (Belopria) zonatus; Michener, 1965. Bull. Amer. Mus. Nat. Hist. 130: 41. - McGinley, 1981. Univ. Calif. Pub. Ent. 91: 77.

Leioproctus (Perditomorpha) zonatus; Michener, 1989. Univ. Kansas Sci. Bull. 53 (11): 635, 638.

Taxonomia. McGinley, 1981: 120-168 (análise fenética e cladística, com base na larva). - Michener, 1989: 635-639 (compara caracteres morfológicos).

Morfologia. McGinley, 1981: 77-80, figs 37-43 (morfologia da larva).

Biologia. Michener \& Lange, 1957. Journ. Kansas Ent. Soc. 30: 76-77; fig. 4 (grande agregação em barrancos no Barigui, Curitiba; descrição dos ninhos, ovos e de pólen). - Michener et al., 1958. Dusenia, Curitiba, 8 (1): 4 (distribuição de ninhos em barrancos terrosos).

\section{Bicolletes Friese}

Bicolletes Friese, 1908. Flora og Fauna 10: 11. - Michener, 1997. Sci. Papers, Nat. Hist. Mus., Univ. Kansas 1: 10.

Espécie-tipo: Bicolletes neotropica Friese, 1908. Designada por Cockerell, 1915. Ann. Mag. Nat. Hist. (8) 15: 342.

Lonchopria (partim); Michener, 1944. Bull. Amer. Mus. Nat. Hist. 82: 237.

Leioproctus (Bicolletes); Michener, 1964. Systematic Zool. 13 (4): 187. - Michener, 1965. Bull. Amer. Mus. Nat. Hist. 130: 41.

Leioproctus (Perditomorpha) (partim); Michener, 1989. Univ. Kansas Sci. Bull. 53 (11): 635- 640.

Taxonomia. Michener, 1944: 237 (Lonchopria [=Bicolletes e Halictandrena [sic]). - Moure, 1951. Dusenia, Curitiba, 2 (3): 197-198 (redescreveu Bicolletes e colocou como sinônimos Pasiphae Spinola, 1851 (partim) e Drachyglossula [sic] Hedicke, 1933). - Moure, 1954. Dusenia, Curitiba, 5 (3/4): 170-171 (redescrição do gênero). - Michener, 1989: 635 (sinônimo de Perditomorpha).

abdominalis (Joergensen). nom. rev. ARgentinA, Mendoza, Chacras de Coria (localidade tipo); Catamarca.

Pasiphae abdominalis Joergensen, 1912. Zool. Jahrb., Abt. Syst. 32: 104. Síntipos fêmeas no MLP.

Bicolletes abdominalis; Moure, 1954. Dusenia, Curitiba, 5 (3/4): 171.

Leioproctus (Bicolletes) abdominalis; Michener, 1964. Systematic Zool. 13 (4): 187.

Leioproctus (Bicolletes) abdominis Michener, 1965. Bull. Amer. Mus. Nat. Hist. 130: 41 (nom. n. para Pasiphae abdominalis Ducke [sic], 1912 non Parocolletes abdominalis Smith, 1879, um Goniocolletes) [sub-gênero de Leioproctus]. 
Leioproctus (Perditomorpha) abdominis; Michener, 1989. Univ. Kansas Sci. Bull. 53 (11): 638.

Taxonomia. Joergensen, 1912: 104 (descreveu somente a fêmea sem indicar holótipo). - Ducke, 1912: 77 (citou Pasiphae abdominalis Joergensen). - Moure, 1954: 190 (figuras da maxila, sétimo esterno e genitália: 3a, 3b e 3c). - Michener, 1964: 187 (sugeriu que Pasiphae abdominalis deveria ser renomeada).

Biologia. Joergensen, 1912: 104 (em Sphaeralcea bonariensis e Larrea divaricata).

alismatis (Ducke) comb.n. BRASIL, Ceará: Quixadá (localidade tipo).

Panurginus alismatis Ducke, 1908. Rev. d'Ent., Caen 27: 66. Lectótipo e lectoalótipo no MPEG. Paralectótipos fêmea e macho no BMNH.

Pasiphae alismatis; Ducke, 1912. Zool. Jahrb., Abt. Syst. 34: 79.

Leioproctus (Bicolletes) alismatis; Michener, 1964. Systematic Zool. 13 (4): 187.

Leioproctus (Perditomorpha) alismatis; Michener, 1989. Univ. Kansas Sci. Bull. 53 (11): 638.

Taxonomia. Nascimento, 1979. Bol. Mus. Paraense Emílio Goeldi, Zoologia 98: 7-8 (designou lectótipo e lectoalótipo por indicação de Moure \& Michener, sem referir-se ao sexo dos exemplares). - Obrecht \& Huber, 1993. Jahrb. Naturhist. Mus. Bern 11: 168 (repetiu informações de Nascimento, 1979).

Biologia. Ducke, 1908: 66 (em flores de Alisma sp., em julho). - Ducke, 1910. Rev. Trim. Inst. Ceará 24: 45 (flores de Alisma (Echinodorus) sp.).

andina (Herbst) comb.n. CHILE, Aconcágua (localidade tipo); Santiago.

Pasiphae andina Herbst, 1923. Rev. Chilena Hist. Nat. 27: 78. Síntipos fêmea e macho no MNHU.

Leioproctus (Bicolletes) andina; Michener, 1964. Systematic Zool. 13 (4): 187.

Leioproctus (Perditomorpha) andinus; Michener, 1989. Univ. Kansas Sci. Bull. 53 (11): 638.

Biologia. Herbst, 1923. Rev. Chilena Hist. Nat. 27: 80 (numerosos exemplares em dezembro na Cordilheira dos Andes, 1. 800-2. 500 m, nas Províncias de Aconcágua e Santiago, sobre flores de Gymnophytum polycephalum).

atacama (Toro \& Rojas) comb.n. CHILE, Atacama: Castilla (localidade tipo).

Leioproctus (Bicolletes) atacama Toro \& Rojas, 1970. An. Mus. Hist. Nat.

Valparaíso 3: 103-105, fig. 5. Holótipo fêmea na CTV.

Leioproctus (Perditomorpha) atacama; Michener, 1989. Univ. Kansas Sci. Bull. 53 (11): 638.

basirufa (Schrottky) comb.n. Argentina, Santa Fé (localidade tipo).

Pasiphae basirufa Schrottky, 1920. Rev. Mus. Paulista 12 (2): 226. Holótipo macho não localizado, provavelmente na coleção Schrottky.

Leioproctus (Perditomorpha) basirufa; Michener, 1989. Univ. Kansas Sci.

Bull. 53 (11): 638.

Taxonomia. Schrottky, 1920: 226 (descreveu o macho). 
bicellularis (Ducke) comb.n. BRASIL, Ceará: Caridade (localidade tipo).

Lonchopria bicellularis Ducke, 1910. Rev. d'Ent., Caen, 28: 81. Lectótipo

e lectoalótipo no MPEG; paralectótipos fêmea e macho no MNHP.

Pasiphae bicellularis; Ducke, 1912: Zool. Jahrb., Abt. Syst. 34: 78.

Leioproctus (Bicolletes) bicellularis; Michener, 1965. Bull. Amer. Mus.

Nat. Hist. 130: 41.

Leioproctus (Perditomorpha) bicellularis; Michener, 1989. Univ. Kansas Sci. Bull. 53 (11): 638.

Taxonomia. Ducke, 1910: 81 (comparou com cearensis, jenseni e joergenseni). - Nascimento, 1979. Bol. Mus. Paraense Emílio Goeldi, Zoologia 98: 6 (designou lectótipo e lectoalótipo por indicação de Moure \& Michener, sem dados sobre o sexo dos mesmos). - Michener, 1989: 639 (listou como Perditomorpha, mas com dúvidas sobre a posição correta desta espécie; comparou com Nomiocolletes, L. (Perditomorpha) eulonchopriodes e L. (P.) leucostomus.

Biologia. Ducke, 1910: 81 (ocorrência no fim de abril e começo de maio).

chrysostoma (Cockerell). ARGENTINA, Santa Fé: Carcarañá (localidade tipo)

Pasiphae chrysostoma Cockerell, 1917. Ann. Mag. Nat. Hist. (8) 20: 438-439. Holótipo macho no USNM.

Bicolletes chrysostoma; Moure, 1954. Dusenia, Curitiba, 5 (3/4): 183.

Leioproctus (Perditomorpha) chrysostomus; Michener, 1989. Univ. Kansas

Sci. Bull. 53 (11): 635, 638.

coloratipes (Cockerell). ARGENTINA, Misiones, Loreto (localidade tipo).

Pasiphae coloratipes Cockerell, 1933. Ann. Mag. Nat. Hist. (10) 11: 461.

Dois síntipos machos no USNM e no BMNH.

Bicolletes colaratipes [sic]; Moure, 1954. Dusenia, Curitiba, 5 (3/4): 183

(comentou que o espécime descrito por Cockerell seria uma fêmea).

Leioproctus (Perditomorpha) coloratipes; Michener, 1989. Univ. Kansas

Sci. Bull. 53 (11): 638.

decolorata (Ducke) comb.n. BRASIL, Ceará: Baturité (localidade tipo), Caridade.

Panurginus decoloratus Ducke, 1908. Rev. d'Ent., Caen, 27: 66. Lectótipo

e lectoalótipo no MPEG; paralectótipos fêmea e macho no BMNH.

Pasiphae decolorata; Ducke, 1912. Zool. Jahrb., Abt. Syst. 34: 79.

Leioproctus (Perditomorpha) decoloratus; Michener, 1989. Univ. Kansas

Sci. Bull. 53 (11): 638.

Taxonomia. Nascimento, 1979. Bol. Mus. Paraense Emílio Goeldi, Zoologia 98: 8 (designou lectótipo e lectoalótipo por indicação de Moure \& Michener, sem referir-se ao sexo dos exemplares). - Obrecht \& Huber, 1993. Jahrb. Naturhist. Mus. Bern 11: 168 (informações sobre os tipos).

Biologia. Ducke, 1908: 66 (em Baturité, em Hyptis suaveolens; na Serra de Baturité, em Borreria verticillata; comum até $700 \mathrm{~m}$, nos meses de junho e julho, na serra até agosto). - Ducke, 1910. Rev. d'Ent., Caen, 28: 83 (em Caridade nos meses de abril e maio). 
erithrogaster (Toro \& Rojas) comb.n. CHILE, Coquimbo: Los Fierros (localidade tipo).

Leoiproctus [sic] (Bicolletes) erithrogaster Toro \& Rojas, 1970. An. Mus. Nac. Hist. Nat., Valparaíso, 3: 105-108, fig. 6. Holótipo fêmea e três parátipos fềmeas na CTV; parátipo fêmea no UCVC; parátipo fêmea no IZUC; três parátipos fêmeas no MHNS.

Leioproctus (Perditomorpha) erithrogaster; Michener, 1989. Univ. Kansas

Sci. Bull. 53 (11): 638, 639.

Taxonomia. Toro \& Rojas, 1970: 108 (discutem sua inclusão em Edwyniana ou Bicolletes).

eulonchopriodes (Michener) comb.n. ARGENTINA, Salta: Pocitos (localidade tipo).

Leioproctus (Perditomorpha) eulonchopriodes Michener, 1989. Univ. Kansas Sci. Bull. 53 (11): 688-689; figs 2 a-f. Holótipo macho no SEMK.

Taxonomia. Michener, 1989: 636, 638, 640, 688-689 (comparação).

fasciata (Schrottky) comb.n. ARGENTINA, Santa Fé (localidade tipo).

Pasiphae fasciata Schrottky, 1920. Rev. Mus. Paulista 12 (2): 224. Síntipos fêmea e macho.

Leioproctus (Perditomorpha) fasciatus; Michener, 1989. Univ. Kansas Sci. Bull. 53 (11): 638.

ferruginea Moure. ARGENTINA, La Rioja: Santa Rosa (localidade tipo).

Bicolletes ferruginea Moure, 1954. Dusenia, Curitiba, 5 (3/4): 174-176. Holótipo fêmea no DZUP.

Leioproctus (Perditomorpha) ferrugineus; Michener, 1989. Univ. Kansas Sci. Bull. 53 (11): 638.

flavitarsus (Toro) comb.n. CHILE, Atacama: Travesía (localidade tipo).

Leioproctus flavitarsus Toro, 1973. An. Mus. Hist. Nat., Valparaíso, 6: 203-207; figs 118-126. Holótipo fêmea e alótipo na CTV. Parátipos 55 machos e 18 fêmeas no UCVC, AMNH, MHNS, SEMK e CTV.

Leioproctus (Perditomorpha) flavitarsus; Michener, 1989. Univ. Kansas Sci. Bull. 53 (11): 635, 638.

Morfologia. Michener, 1989: 635 (mandíbula com dente pré-apical em ambos os sexos).

franki Friese. ARgENTINA, Buenos Aires (localidade tipo).

Bicolletes franki Friese, 1908. Flora og Fauna 10: 12. Holótipo macho no MNHU.

Pasiphae franki; Ducke, 1912. Zool. Jahrb., Abt. Syst. 34: 78-79.

Leioproctus (Bicolletes) franki; Michener, 1964. Systematic Zool. 13 (4): 187.

Leioproctus (Perditomorpha) franki; Michener, 1989. Univ. Kansas Sci. Bull. 53 (11): 638.

Biologia. Wittmann \& Schlindwein, 1995. Biociências, Porto Alegre, 3 (2): 19-34 (fêmeas oligoléticas em flores de Cajophora arechavaletae e com pólen de 
Glechon (Lamiaceae) e Croton (Euphorbiaceae). - Schlindwein, 1995. Wildbienen und ihre Trachtpflanzen..., Stuttgart: 88 (lista exemplares desta espécies coletados em flores de: Schinus molle (Anacardiaceae), Croton cf. gnaphalii, C. thermarum Euphorbiaceae), Cunila incisa, Glechon thymoides (Lamiaceae), Nothoscordum inodorum (Liliaceae), Blumenbachia insignis, Cajophora arechavaletae (Loasaceae).

friesei (Joergensen) comb.n. ARGENTINA, Mendoza Chacras de Coria (localidade tipo).

Pasiphae friesei Joergensen, 1912. Zool. Jahrb., Abt. Syst. 32: 102. Síntipos fêmea e macho no MLP.

Leioproctus (Bicolletes) friesei; Michener, 1964. Systematic Zool. 13 (4): 187.

Leioproctus (Perditomorpha) friesei; Michener, 1989. Univ. Kansas Sci. Bull. 53 (11): 638.

Taxonomia. Ducke, 1912: 77 (listou Pasiphae friesei).

Biologia. Joergensen, 1912: 103 (observou em Sphaeralcea bonariensis, Cristaria loasifolia, Flaveria contrayerba).

iheringi (Schrottky). BRASIL, São Paulo: São Paulo, Ypiranga (localidade tipo); Rio de Janeiro, (Tijuca) (450-600m).

Pasiphae iheringi Schrottky, 1910. Ent. Rundsch. 27: 56. Holótipo fêmea.

Pasiphae cestri Ducke, 1912. Zool. Jahrb., Abt. Syst. 34: 77-78. Lectótipo, lectoalótipo e paralectótipos no MPEG; paralectótipos fêmea e macho no MNHU.

Bicolletes iheringi; Moure, 1951. Dusenia, Curitiba, 2 (3): 198.

Leioproctus (Perditomorpha) iheringi; Michener, 1989. Univ. Kansas Sci. Bull. 53 (11): 635, 636, 638.

Taxonomia. Moure, 1951: 198 (iheringi = cestri Ducke). - Moure, 1954. Dusenia, Curitiba, 5 (3/4): 189 e figs 5 a, b, c (esternos não expostos e genitália). - Nascimento, 1979. Bol. Mus. Paraense Emílio Goeldi, Zoologia 98: 8 (designou lectótipo, lectoalótipo e paralectótipos de $P$. cestri por indicação de Moure \& Michener, sem se referir ao sexo dos exemplares).

Biologia. Luederwaldt, 1910. Ztschr. wiss. Insektenbiologie 6: 297 (biologia). - Ducke, 1912: 78 (em flores de Cestrum, em novembro). - Michener \& Lange, 1957. Journ. Kansas Ent. Soc. 30 (2): 77 (descrição de ninhos). - Michener et al., 1958, Dusenia, Curitiba, 8 (1): 4, 9 (distribuição de ninhos em barrancos em Curitiba).

leucostoma (Cockerell). ARgEnTINA, Santa Fé, Carcarañá (localidade tipo); Tucumán, S. Pedro Colalao.

Pasiphae leucostoma Cockerell, 1917. Ann. Mag. Nat. Hist. (8) 20: 439. Holótipo macho no USNM.

Bicolletes leucostoma; Moure, 1954. Dusenia, Curitiba, 5 (3/4): 178-180.

Leioproctus (Perditomorpha) leucostomus; Michener, 1989. Univ. Kansas

Sci. Bull. 53 (11): 635, 636 fig. g, 638, 639. 
Taxonomia. Moure, 1954: 178-180 (redescrição do macho). - Michener, 1989: 639 (comentou sua posição entre Perditomorpha e Bicolletes).

longipes (Joergensen) comb.n. ARGENTINA, Mendoza, Chacras de Coria (localidade tipo).

Pasiphae longipes Joergensen, 1912. Zool. Jahrb., Abt. Syst. 32: 101-102. Síntipos fêmea e macho no MLP.

Leioproctus (Perditomorpha) longipes; Michener, 1989. Univ. Kansas Sci. Bull. 53 (11): 638.

Biologia. Joergensen, 1912: 102 (ocorrência entre 26 de outubro e fins de fevereiro em Larrea divaricata).

lucidula (Cockerell) comb.n. ARGENTINA, Misiones: Loreto (localidade tipo).

Pasiphae lucidula Cockerell, 1933. Ann. Mag. Nat. Hist. (10) 11: 461-462. Síntipos machos no USNM e BMNH.

Leioproctus (Perditomorpha) lucidulus; Michener, 1989. Univ. Kansas Sci. Bull. 53 (11): 638.

moerens (Vachal) comb.n. ARGENTINA, Mendoza (localidade tipo).

Pasiphae moerens Vachal, 1909. Rev. d' Ent., Caen, 28: 39. Holótipo fềmea no MNHP. Leioproctus (Perditomorpha) moerens; Michener, 1989. Univ. Kansas Sci. Bull. 53 (11): 638.

neotropica Friese. ARgENTINA, Mendoza (localidade tipo).

Bicolletes neotropica Friese, 1908. Flora og Fauna 10: 11-12. Síntipos macho e fêmea no MNHU.

Pasiphae neotropica; Joergensen, 1912. An. Mus. Nac., Buenos Aires, 22: 301

Leioproctus (Perditomorpha) neotropicus; Michener, 1989. Univ. Kansas Sci. Bull. 53 (11): 638, 639.

Taxonomia. Cockerell, 1915. Ann. Mag. Nat. Hist. (8) 15: 342 (designação subsequente como espécie-tipo de Bicolletes). - Moure, 1951, Dusenia, Curitiba, 2 (3): 197-198 (redescrição). - Moure, 1954, Dusenia, Curitiba, 5 (3/4): 170 (redescrição). - Nascimento, 1979. Bol. Mus. Paraense Emílio Goeldi, Zoologia 98: 3 (citou como holótipo fêmea de B. neotropicus Friese, 1908, um exemplar de Tucumán, $2000 \mathrm{~m}$ da coleção do MPEG. Friese descreveu macho e fêmea desta espécie, de Mendoza, não designou holótipo).

Biologia. Friese, 1908: 12 (agrupados em grande número, entre 5 de dezembro e 10 de janeiro, em Mendoza). - Joergensen, 1909. Deutsch. Ent. Ztschr. : 56 (em Grindelia pulchella; G. speciosa; Baccharis serrulata, B. salicifolia; B. subulata; B. pingrea; B. juncea; B. effusa, B. sagitallis; Tessaria absinthoides; Senecio pinnatus; S. mendocinus; S. albicaulis; S. stipellatus; Taraxacum officinale; Solidago linearifolia; Cynara cardunculus; Ximenedia microptera; Bidens leucantha; Achyrophorus glaucus; Hysterionica jasionoides; Parthenium hysterionoides; Aster linifolius; Triscis papillosa; Cirsium lanceolatum; Flaveria contrahyerba; Centaurea melitensis; Pascalia glauca (Compositae [Asteraceae]); Caesalpinia precox; Prosopis alpataco; P. strombulifera; P. campestris; Acacia furcata (Legu- 
minosae); Sphaeralcea bonariensis; S. mendocina; S. collina; Malva parviflora; M. rotundifolia; Sida leprosa; Abutilon mendocinum (Malvaceae); Clematis hilarii; Tamarix africana; Convolvulus arvensis; C. hermanniae; Cuscuta racemosa (Convolvulaceae). - Joergensen, 1912: 301 (comum desde 29 de outubro até 11 de abril; flores como citadas acima).

nigriceps (Friese) comb.n. PARAGUAI, Villa Rica (localidade tipo).

Panurginus nigriceps Friese, 1916. Stett. Ent. Ztg. 77: 172. Síntipo macho no MNHU, examinado por J. S. Moure.

Taxonomia. Friese, 1916: 172 (não indicou o número de exemplares).

orientalis (Vachal) comb.n. ARgENTINA, Tucumán: Santa Ana, 350m (localidade tipo). CHILE, Antofagasta.

Chilicola (?) orientalis Vachal, 1904. Rev. d'Ent., Caen, 23: 24. " mauvais état, que je suppose être mâles". Síntipos no MNHP.

Pasiphae orientalis; Vachal, 1909. Rev. d'Ent., Caen, 28: 39.

Taxonomia. Vachal, 1909: 39 (comentou que a espécie não era do gênero Chilicola, mas do gênero Pasiphae e referiu-se a um exemplar de Antofagasta, Chile). - Joergensen, 1912. An. Mus. Nac., Buenos Aires, 22: 301 (comparou com Pasiphae longipes). - Cockerell, 1926. Ann. Mag. Nat. Hist. (9) 17: 220 (colocou em dúvida a identificação do exemplar de Antofagasta, citado por Vachal, 1909).

pampeana Urban. BRAsIL, Rio Grande do Sul, Caçapava do Sul (localidade tipo), Guaritas, Minas de Camaquã.

Bicolletes pampeana Urban, 1995. Revta bras. Zool. 12 (2): 402-403. Holótipo macho no DZUP; parátipos no DZUP e na UCRS.

Taxonomia. Urban, 1995: 403 (próxima a Bicolletes franki). - Schlindwein, 1995. Wildbienen und ihre Trachtpflanzen..., Stuttgart: 88 (cita B. pampeana Urban \& Moure in litt.).

Biologia. Schlindwein, 1995. Wildbienen und ihre Trachtpflanzen..., Stuttgart: 88 (em Senecio leptolobus, Glechon thymoides, Blumenbachia insignis, Cajophora arechavaletae).

paraguayensis (Schrottky). PARAGUAI, Villa Encarnación (localidade tipo).

Perditomorpha paraguayensis Schrottky, 1907. An. Ci. Paraguayos 1 (7): 44, 45- 47. Síntipos fêmea e macho.

Bicolletes paraguayensis; Moure, 1951. Dusenia, Curitiba, 2 (3): 198.

Leioproctus (Perditomorpha) paraguayensis; Michener, 1989. Univ. Kansas Sci. Bull. 53 (11): 638.

Taxonomia. Moure, 1951: 191 (nota).

Biologia. Schrottky, 1907: 47 (coletado em outubro, sobre Oxalis com flores rosadas).

penai (Toro \& Rojas) comb.n. CHILE, Malleco, Lago Galletué (localidade tipo).

Leioproctus [(Bicolletes)] penai Toro \& Rojas, 1970. An. Mus. Hist. Nat.

Valparaíso 3: 90-94, fig. 2. Holótipo macho, alótipo e parátipos no MHNS; parátipos no UCVC, SEMK e CTV. 
Leioproctus (Perditomorpha) penai; Michener, 1989. Univ. Kansas Sci. Bull. 53 (11): 638.

Leioproctus penal [sic]; Toro \& Rojas, 1970: 109.

perezi (Toro \& Rojas) comb.n. CHILE, Coquimbo: Peñuelas (localidade tipo), Choros, Los Fierros

Leioproctus [(Bicolletes)] perezi Toro \& Rojas, 1970. An. Mus. Hist. Nat. Valparaíso 3: 95-99. Holótipo macho, alótipo e parátipos no MHNS; parátipos no UCVC, SEMK, CTV e IZUC.

Leioproctus (Perditomorpha) perezi; Michener, 1989. Univ. Kansas Sci. Bull. 53 (11): 638.

Morfologia. Toro \& Rojas, 1970: 98 (variação de tamanho, coloração e forma do oitavo esterno dos machos).

peruviana (Cockerell). PERU, Tingo [Maria?] (localidade tipo); Caraz, 2800m.

Pasiphae peruviana Cockerell, 1926. Ann. Mag. Nat. Hist. (9) 17: 220. Holótipo macho no USNM.

Brachyglossula peruviana; Moure, 1944. Bol. Mus. Hist. Nat. "Javier Prado" 8: 74.

Bicolletes peruviana; Moure, 1954. Dusenia, Curitiba, 5(3/4): 187-188, figs 4 a-c.

Leioproctus (Perditomorpha) peruvianus; Michener, 1989. Univ. Kansas Sci. Bull. 53 (11): 638.

Taxonomia. Cockerell, 1926: 220 (comparou com P. rufiventris e P. tristis). pharcidodes Moure. PERU, Ayacucho 2700m (localidade tipo), Huancuayo.

Bicolletes pharcidodes Moure, 1954. Dusenia, Curitiba, 5 (3/4): 183-186, figs 6 a-c. Síntipos: uma fêmea e sete machos no DZUP e no MJP.

Leioproctus (Perditomorpha) pharcidodes; Michener, 1989. Univ. Kansas

Sci. Bull. 53 (11): 638.

Taxonomia. Moure, 1954: 186 (comparou com B. leucostoma e B. peruvia$n a)$.

pseudozonata Moure. ARgEntinA, Tucumán, San Pedro Colalao (localidade tipo)

Bicolletes pseudozonata Moure, 1954. Dusenia, Curitiba, 5 (3/4): 180-183. Síntipos fêmea e macho no DZUP.

Leioproctus (Perditomorpha) pseudozonatus; Michener, 1989. Univ. Kansas Sci. Bull. 53 (11): 638.

Taxonomia. Moure, 1954: 183 (comparou com B. leucostoma, B. abdominalis stilborhina e $B$. chrysostoma)

rubra (Toro \& Rojas) comb.n. CHILE, La Serena, Las Trancas (localidade tipo); Churqui.

Leioproctus (Bicolletes) ruber Toro \& Rojas, 1970. An. Mus. Hist. Nat. Valparaíso 3: 85-90, figs 1 a-d. Holótipo fêmea e alótipo no MHNS; dois parátipos, fêmea e macho na CTV.

Leioproctus (Perditomorpha) ruber; Michener, 1989. Univ. Kansas Sci. Bull. 53 (11): 635, 636, 638.

Taxonomia. Toro \& Rojas, 1970: 85 (comparou com L. rufiventris). 
rufiventris (Spinola). CHILE, Santiago (localidade tipo). ARGENTINA, Mendoza, Chacras de Cória.

Pasiphae rufiventris Spinola, 1851, in Gay. Hist. Fis. Pol. Chile, Zool. 6: 229. Holótipo fêmea no MIZT.

Bicolletes rufiventris; Moure, 1951. Dusenia, Curitiba, 2 (3): 197, 198.

Leioproctus (Bicolletes) rufiventris; Michener, 1964. Systematic Zool. 13

(4): 187. - Michener, 1965. Bull. Amer. Mus. Nat. Hist. 130: 41.

Leiproctus [sic] (Bicolletes) rufiventris; Toro \& Rojas, 1970. An. Mus. Hist. Nat. Valparaíso 3: 85.

Leioproctus (Perditomorpha) rufiventris; Michener, 1989. Univ. Kansas

Sci. Bull. 53 (11): 638.

Taxonomia. Vachal, 1901. An. Soc. Ent. Fr. 70: 79 (nota morfológica). Cockerell, 1939. Amer. Mus. Novitates 1046: 1 (comparou com P. perornata).

Biologia. Joergensen, 1912. An. Mus. Nac., Buenos Aires, 22: 302 (comuns em Chacras de Cória desde 26 de outubro até 5 de dezembro em Sphaeralcea bonariensis e Cristaria loasifolia). - Janvier, 1933. Ann. Sci. Nat., Zool., Paris, (10) 16: 327 (observou espécimens suspensos em pequenos grupos à noite ou individualmente em flores de Loasa).

saltensis Friese. ARGENTINA, Salta (localidade tipo).

Bicolletes saltensis Friese, 1908. Flora og Fauna 10: 12. Síntipos fêmeas no MNHU.

Pasiphae saltensis; Ducke, 1912. Zool. Jahrb., Abt. Syst. 34: 78.

Taxonomia. Friese, 1908: 12 (comparou com B. neotropicus).

seydi Strand. PERU (localidade tipo).

Bicolletes seydi Strand, 1910. Jahrb. Nass. Ver. Nat., Wiesbaden, 63: 8.

Tipo não localizado no MNHU.

Pasiphae seydi; Cockerell, 1939. Amer. Mus. Novit. 1046: 2.

Leioproctus (Perditomorpha) seydi; Michener, 1989. Univ. Kansas Sci.

Bull. 53 (11): 638.

spegazzini (Joergensen). ARgentinA, Mendoza, La Paz (localidade tipo).

Calliopsis (?) spegazzini Joergensen, 1912. Zool. Jahrb., Abt. Syst. 32: 121. Holótipo macho no MLP.

Calliopsis (?) Spegazzinii [sic]; Joergensen, 1912. An. Mus. Nac., Buenos

Aires, 22: 308

Leioproctus (Bicolletes) spegazzinii [sic]; Michener, 1964. Systematic Zool. 13 (4): 187.

Bicolletes spegazzini; Shinn, 1967. Univ. Kansas Sci. Bull. 46 (21): 895.

Leioproctus (Perditomorpha) spegazzinii [sic]; Michener, 1989. Univ. Kansas Sci. Bull. 53 (11): 638.

Taxonomia. Joergensen, 1912: 308 (citou o macho de Mendoza).

sticta Moure. ArgentinA, Tucumán, San Pedro Colalao (localidade tipo).

Bicolletes sticta Moure, 1954. Dusenia, Curitiba, 5 (3/4): 176. Holótipo fềmea no DZUP. 
Leioproctus (Perditomorpha) stictus; Michener, 1989. Univ. Kansas Sci. Bull. 53 (11): 638.

Taxonomia. Moure, 1954: 176 (comparou com B. saltensis e B. leucostoma). stilborhina Moure. ARgentinA, Catamarca, Catamarca (localidade tipo).

Bicolletes abdominalis stilborhina Moure, 1954. Dusenia, Curitiba, 5 (3/4):

171-174. Síntipos, dois machos e uma fêmea no DZUP.

Leioproctus (Perditomorpha) stilborhinus; Michener, 1989. Univ. Kansas

Sci. Bull. 53 (11): 635, 636, 638.

Taxonomia. Moure, 1954: 174 (comparou com B. neotropica).

wagenknechti (Toro \& Rojas) comb.n.. CHILE, Coquimbo, El Tofo (localidade tipo), Choros.

Leioproctus (Bicolletes) wagenknechti Toro \& Rojas, 1970. An. Mus. Hist.

Nat. Valparaíso 3: 99-103, figs 4 a-d. Holótipo fêmea e alótipo na CTV; parátipos fêmeas e machos no MHNS, UCVC, SEMK; CTV e IZUC.

Leioproctus (Perditomorpha) wagenknechti; Michener, 1989. Univ. Kansas Sci. Bull. 53 (11): 638.

\section{Biglossa Friese}

Biglossa Friese, 1906, Ztschr. Hym. Dipt. 6: 374-380. - Michener, 1997. Sci. Papers, Nat. Hist. Mus., Univ. Kansas 1: 10.

Espécie-tipo: Biglossa thoracica Friese. Designada por Cockerell, 1914. Journ. N. Y. Ent. Soc. 22: 328. Leioproctus (Biglossa); Michener, 1964. Systematic Zool. 13 (4): 187. - Michener, 1965. Bull. Amer. Mus. Nat. Hist. 130: 41.

Lonchopria (Biglossa) (partim); Michener, 1989. Univ. Kansas Sci. Bull. 53 (11): 673.

Taxonomia. Michener, 1989: 676 (comentou que a maioria das espécies de Lonchopria (Biglossa) poderia ser colocada no sub-gênero Biglossidia, isso resultaria num sub-gênero monotípico para $L$. thoracica, que apresenta alguns caracteres apomórficos, entre outros a mandíbula do macho tridentada).

thoracica Friese. ARgENTINA, Salta, 1200m-2500m (localidade tipo); Tucumán; Mendoza, Chacras de Coria.

Biglossa thoracica Friese, 1906. Ztschr. Hym. Dipt. 6: 376. Síntipos: 2 machos e 12 fêmeas. Depositados no MNHU, USNM, AMNH.

Lonchopria (Biglossa) thoracica; Michener, 1989. Univ. Kansas Sci. Bull. 53 (11): 673- 676, 678.

Taxonomia. Friese, 1908. Flora og Fauna 10: 13-14 (chave, redescrição). - Moure, 1948. Rev. Ent., Rio de Janeiro, 19 (1/2): 315 (comparação com Biglossidia). - Michener, 1989: 673-678, figs a-i.

Biologia. Jensen-Haarup, 1908. Flora og Fauna 10: 98 (em flores de Physalis, Solanaceae, em grande número em Chacras de Coria, de fevereiro a abril). - Joergensen, 1912. An. Mus. Nac., Buenos Aires, 22: 302 (abundantes em toda a Província de Mendoza, de 26. X a 12. VI, em Baccharis serrulata, B. salicifolia, $B$. subulata, Bidens leucantha, Senecio pinnatus, Heterothalamus spartioides, Lycium gracile, L. chilense, L. argentinum, Physalis viscosa, Solanum elaeagnifolium, $S$. 
atriplicifolium, Grobowskya obtusa, Prosopis alpataco, P. campestris, Acacia furcata, Glycyrrhiza astragalina, Medicago sativa, Larrea divaricata, Bulnesia retama, Clematis hilarii).

\section{Biglossidia Moure}

Biglossidia Moure, 1948. Rev. Ent., Rio de Janeiro, 19 (1/2): 313. - Michener, 1997. Sci. Papers, Nat. Hist. Mus., Univ. Kansas 1: 10.

Espécie-tipo: Biglossa chalybaea Friese, 1906. Designação original.

Leioproctus (Biglossidia); Michener, 1964. Systematic Zool. 13 (4): 187. - Michener, 1965. Bull. Amer. Mus. Nat. Hist. 130: 41.

Lonchopria (Biglossa) (partim); Michener, 1989. Univ. Kansas Sci. Bull. 53 (11): 673.

Taxonomia. Moure, 1948: 313-315 (comparação com Biglossa, Lonchopria e Nomiocolletes). - Moure 1949, Rev. Ent., Rio de Janeiro, 20 (1/3): 448-449 (chave). - Michener, 1989: 673-678 (considerou Biglossidia como sinônimo de Biglossa).

aenea (Friese) ARGENTINA, Salta, 2500m (localidade tipo).

Biglossa aenea Friese, 1906. Ztschr. Hym. Dipt. 6: 379. Tipo macho depositado no MNHU.

Biglossidia aenea; Moure, 1949. Rev. Ent., Rio de Janeiro, 20 (1/3): 445-447.

Leioproctus (Biglossidia) aenea; Michener, 1964. Systematic Zool. 13 (4): 187.

Lonchopria (Biglossa) aenea; Michener, 1989. Univ. Kansas Sci. Bull. 53 (11): 676, 678 .

Taxonomia. Friese, 1906: 375 (chave). - Moure, 1948, Rev. Ent., Rio de Janeiro, 19 (1/2): 316 (identificou e descreveu como Biglossidia aenea uma fêmea, a qual no ano seguinte descreveu como B. deceptrix). - Moure, 1949: 445-447 (redescreveu macho e fêmea). - Michener, 1989: 676, 678 (citou a espécie).

alopex (Cockerell) comb.n. BolíviA, La Paz (localidade tipo).

Lonchopria alopex Cockerell, 1917. Ann. Mag. Nat. Hist. (8) 19: 480. Holótipo no USNM.

Leioproctus (Lonchopria) alopex; Michener, 1964. Systematic Zool. 13 (4): 187.

Lonchopria (Biglossa) alopex; Michener, 1989. Univ. Kansas Sci. Bull. 53 (11): 676.

chalybaea (Friese). ARgENTINA, Salta, 1200-2500m (localidade tipo); Tucumán, Tacanas; Catamarca; Mendoza.

Biglossa chalybaea Friese, 1906. Ztschr. Hym. Dipt. 6: 378. Síntipo fêmea no MNHU.

Biglossa armata Friese, 1906. Ztschr. Hym. Dipt. 6: 379. Holótipo macho no MNHU.

Lonchopria armata; Ducke, 1910. Rev. d'Ent., Caen, 28: 81.

Lonchopria chalybea [sic]; Schrottky, 1909. An. Soc. Ci. Arg. 68: 252. Ducke, 1912. Zool. Jahrb., Abt. Syst. 34: 80. 
Lonchopria chalybaea; Schrottky, 1913. An. Soc. Ci. Arg. 75: 237.

Biglossidia chalybaea; Moure, 1948. Rev. Ent., Rio de Janeiro, 19 (1/2):

315.

Leioproctus (Biglossidia) armata; Michener, 1964. Systematic Zool. 13 (4):

187.

Leioproctus (Biglossidia) chalybaea; Michener, 1964. Systematic Zool. 13 (4): 187.

Lonchopria (Biglossa) chalybaea; Michener, 1989. Univ. Kansas Sci. Bull. 53 (11): 673-676, 678; figs 25 a-h.

Taxonomia. Friese, 1908. Flora og Fauna 10: 16 (repetição da descrição original). - Ducke, 1910. Rev. d'Ent., Caen, 28: 81 (nota comparativa de Lonchopria armata e L. cearensis). - Cockerell, 1914. Journ. N. Y. Ent. Soc. 22: 328 (nota comparativa entre L. chalybaea e L. thoracica). - Moure, 1949, Rev. Ent., Rio de Janeiro, 20 (1/3): 448-449 (chave). - Michener, 1989: 673-678, figs 25 a-h. (comparações).

Biologia. Jensen-Haarup, 1908. Flora og Fauna 8: 100 (em Baccharis marginalis var. coerulescens e Parchenium hysterophorus no meio do verão).

deceptrix Moure. ARGENTINA, Tucumán, Tacanas (localidade tipo).

Biglossidia aenea; Moure, 1948. Rev. Ent., Rio de Janeiro, 19 (1/2): 316; non Biglossa aenea Friese, 1906.

Biglossidia deceptrix Moure, 1949. Rev. Ent., Rio de Janeiro, 20 (1/3): 447-449. Holótipo, alótipo e quatro parátipos no IML, sete parátipos no DZUP, sem indicação do sexo do holótipo.

Leioproctus (Biglossidia) deceptrix; Michener, 1964. Systematic Zool. 13 (4): 187.

Lonchopria (Biglossa) deceptrix; Michener, 1989. Univ. Kansas Sci. Bull. 53 (11): 676, 678.

Taxonomia. Moure, 1948: 316-317 (descreveu uma fêmea desta espécie como Biglossidia aenea Friese, 1906). - Michener, 1989: 676, 678 (citou a espécie). inca (Cockerell) comb.n. PERU, Matucana (localidade tipo).

Lonchopria inca Cockerell, 1914. Journ. N. Y. Ent. Soc. 22: 327-328. Síntipos: dois machos no USNM.

Lonchopria (Biglossa) inca; Michener, 1989. Univ. Kansas Sci. Bull. 53 (11): 674, 676, 678 .

longicornis (Michener) comb.n. ARGENTINA, Catamarca, Ruta 4, cinco km ao sul de El Rodeo, 1450m (localidade tipo); Andalgalá.

Lonchopria (Biglossa) longicornis Michener, 1989. Univ. Kansas Sci. Bull. 53 (11): 700-702; figs 27 a-h. Holótipo macho, alótipo e parátipos no SEMK, parátipos no MBR e em outras coleções conforme Michener, 1989: 702 .

Taxonomia. Michener, 1989: 674, 675, 676, 678 (compara com outras espécies). 
robertsi (Michener) comb.n. ARGENTINA, Tucumán, $5 \mathrm{~km}$ a leste de Amaicha, 2300m (localidade tipo); Catamarca, Andalgalá, Minas Capillilas, El Pucará, Joyango, Cuesta de la Chilca, Londres; Chubut, Pen. Valdez.

Lonchopria (Biglossa) robertsi Michener, 1989. Univ. Kansas Sci. Bull. 53 (11): 702- 703; figs 28 a-j; 31 g. Holótipo macho e alótipo fêmea no SEMK; parátipos no SEMK, DZUP, MBR, BMNH e outras coleções conforme Michener, 1989: 703.

Biologia. Michener, 1989: 703 (em flores de Larrea divaricata, Larrea cuneifolia, Bulnesia retama e Prosopis nigra).

\section{Brachyglossula Hedicke}

Brachyglossa Friese, 1922. Zool. Jahrb., Abt. Syst. 45: 577 (nom. praeoc. Boisduval, [1828]. Europ. Lepid. Index, 1829. 33. Lep.).

Brachyglossula Hedicke, 1922. Deutsch. Ent. Ztschr. : 427; nom. n. para Brachyglossa Friese, 1922. - Sandhouse, 1943. Proc. U. S. Natl. Mus. 92: 531. - Michener, 1989. Univ. Kansas Sci. Bull. 53 (11): 663. - Michener, 1997. Sci. Papers, Nat. Hist. Mus., Univ. Kansas 1: 11.

Espécie-tipo: Brachyglossa rufocaerulea Friese. Monotipia.

Leioproctus (Brachyglossula); Michener, 1964. Systematic Zool. 13 (4): 187. - Michener, 1965. Bull. Amer. Mus. Nat. Hist. 130: 41.

Taxonomia. Sandhouse, 1943: 531 (relacionou Brachyglossa rufocaerulea Friese, 1922 como espécie-tipo de Brachyglossula). - Michener, 1964: 187 (considerou Brachyglossula como sub-gênero de Leioproctus). - Michener, 1989: 663665 (redescreveu o gênero e incluiu quatro espécies).

bouvieri (Vachal). PERU, Cuzco (localidade tipo), Puno. BOLíVIA, Mapiri.

Pasiphae bouvieri Vachal, 1901. Ann. Soc. Ent. Fr. 70: 79. Síntipos: três fêmeas e dois machos depositados no MNHP.

Pasiphae boliviensis Vachal, 1901. Ann. Soc. Ent. Fr. 70: 79. Holótipo fêmea depositado no MNHP. Syn. nov.

Brachyglossa rufocaerulea Friese, 1922, Zool. Jahrb., Abt. Syst. 45: 578580; figs A, B e C. Holótipo macho depositado no MNHU. Syn. nov.

Pasiphae perornata Cockerell, 1939. Amer. Mus. Novitates. 1046: 1-2. Síntipo fêmea depositado no AMNH. Syn. n.

Brachyglossula boliviensis; Michener, 1989. Univ. Kansas Sci. Bull. 53 (11): 665.

Brachyglossula bouvieri; Michener, 1989. Univ. Kansas Sci. Bull. 53 (11): 665; figs 20 a-1.

Brachyglossula personata [sic]; Michener, 1989. Univ. Kansas Sci. Bull. 53 (11): 665.

Brachyglossula rufocoerulea [sic]; Michener, 1989. Univ. Kansas Sci. Bull. 53 (11): 665.

Taxonomia. Ducke, 1912. Zool. Jahrb., Abt. Syst. 34: 77 (citou P. bouvieri e P. boliviensis na listagem de espécies de Pasiphae). - Hedicke, 1933. Mitt. Deutsch. Entom. Ges. 4 (3): 46 (colocou $B$. rufocaerulea na sinonímia de $B$. boliviensis). - Cockerell, 1939. Amer. Mus. Novitates 1046: 1-2 (nota comparativa). - Sandhouse, 1943. Proc. U. S. Natl. Mus. 92: 531 (B. rufocaerulea como espécie-tipo). - Moure, 1944. Bol. Mus. Hist. Nat. "Javier Prado" 8: 74. - Moure, 
1951, Dusenia, Curitiba, 2 (3): 189-193 (nota comparativa). - Michener, 1964: 187. - Michener, 1965: 41.

Biologia. Michener, 1989: 665 (visita flores de Cactaceae).

\section{Cephalocolletes Michener}

Leioproctus (Cephalocolletes) Michener, 1989. Univ. Kansas Sci. Bull. 53 (11): 657-658. - Michener, 1997. Sci. Papers, Nat. Hist. Mus., Univ. Kansas 1: 13.

Espécie-tipo: Biglossa laticeps Friese. Designação original.

Cephalocolletes; Urban, 1995: 397-398.

Taxonomia. Michener, 1989: 657-658; figs 14 a-g, 16 d (comparou com Reedapis, Brachyglossula e Lonchopria (Biglossa). - Urban, 1995: 397-405 (stat. nov. ; espécies novas).

crassipunctata Urban. ARgentinA, Catamarca, Campo de Pucara (localidade tipo).

Cephalocolletes crassipunctata Urban, 1995. Revta. bras. Zool. 12 (2): 401. Holótipo fêmea e parátipos no DZUP.

isabelae Urban. BRASIL, Rio Grande do Sul, Capão da Canoa (localidade tipo); Santa Catarina, Pântano do Sul.

Cephalocolletes isabelae Urban, 1995. Rev. bras. Zool. 12 (2): 398-399;

fig. 1. Holótipo fêmea, alótipo macho e parátipos no DZUP; parátipos na UCRS.

laticeps (Friese). ARgENTINA, Tucumán, 2000m (localidade tipo); La Rioja; Catamarca; Mendoza.

Biglossa laticeps Friese, 1906. Ztschr. Hym. Dipt. 6: 376-377. Síntipo macho depositado no MNHU.

Leioproctus (Cephalocolletes) laticeps; Michener, 1989. Univ. Kansas Sci.

Bull. 53 (11): 657, 658; figs 14 a-i.

Cephalocolletes laticeps; Urban, 1995. Revta. bras. Zool. 12 (2): 397.

Taxonomia. Friese, 1906: 375 (chave).

Biologia. Joergensen, 1912. An. Mus. Nac., Buenos Aires, 22: 302 (machos e fêmeas comuns de novembro a março em Mendoza, em Prosopis alpataco e $P$. campestris, Physalis viscosa e Opuntia sulphurea).

rugata Urban. BRASIL, Rio Grande do Sul: Caçapava do Sul (localidade tipo), Santana da Boa Vista, Canguçu, Bagé, Taim. Argentina, Entre Rios: La Paz.

Cephalocolletes rugata Urban, 1995. Revta. bras. Zool. 12 (2): 400. Holótipo fêmea e parátipos no DZUP; parátipos na UCRS.

Taxonomia. Schlindwein \& Wittmann, 1995. Bradleya 13: 25 -28 (Sarocolletes rugata Urban \& Moure [sic]).

Biologia. Schlindwein \& Wittmann, 1995. Bradleya 13: 25-32 (poliniza flores de Notocactus sellowii e N. succineus; visita flores de Notocactus mammulosus, $N$. ottonis, Opuntia brunneogemmia, O. viridirubra e Gymnocalycium denudatum, Cactaceae; e Monnina cuneata, Polygalaceae; coleta pólen na escopa ventral, 
com cerdas longas não ramificadas, próprias para grãos de pólen grandes). Schlindwein, 1995. Biociências, Porto Alegre, 3 (2): $42-57$ (coleta de pólen de Notocactus mammulosus, $N$. ottonis, $N$. sellowii, $N$. succineus, Opuntia viridirubra e O. brunneogemmia no Rio Grande do Sul). - Schlindwein, 1995. Wildbienen und ihre Trachtpflanzen..., Stuttgart: 89 (em Cactaceae e Polygalaceae).

\section{Chilicolletes Michener, stat. nov.}

Leioproctus (Chilicolletes) Michener, 1989. Univ. Kansas Sci. Bull. 53 (11): 640-641. - Michener, 1997. Sci. Papers, Nat. Hist. Mus., Univ. Kansas 1: 15.

Espécie-tipo: Leioproctus delahozii Toro. Designação original.

Taxonomia. Michener, 1989: 640-641 (comparou com Perditomorpha).

delahozii (Toro) comb.n. CHILE, Coquimbo: El Tofo (localidade tipo), La Serena, Copiapó.

Leioproctus delahozii Toro, 1973, An. Mus. Nac., Chile, 6: 210-212; figs

127-135. Holótipo macho e alótipo na CTV; parátipos: 37 machos e 38 fêmeas no MHNS, AMNH, UCVC e SEMK.

Leioproctus (Chilicolletes) delahozii; Michener, 1989. Univ. Kansas Sci.

Bull. 53 (11): 640, 641; figs 4 a-c, 10 c.

Taxonomia. Toro, 1973: 210 (considerou semelhante a L. flavitarsus). Toro, 1986. Acta Ent. Chilena 13: 122 (distribuição geográfica).

\section{Ctenosibyne Moure, stat. nov.}

Lonchopria (Ctenosibyne) Moure, 1956. Dusenia, Curitiba, 7 (6): 311-313. - Michener, 1989. Univ. Kansas Sci. Bull. 53 (11): 681. - Michener, 1997. Sci. Papers, Nat. Hist. Mus., Univ. Kansas 1: 18 .

Espécie-tipo: Lonchopria (Ctenosibyne) cingulata Moure, 1956. Designação original.

Leioproctus (Ctenosibyne); Michener, 1964. Systematic Zool. 13 (4): 187. - Michener, 1965. Bull. Amer. Mus. Nat. Hist. 130: 41.

Taxonomia. Michener, 1989: 681-682, figs $30 \mathrm{a}-\mathrm{g}$ (redescreveu o sub-gênero e o considerou intermediário entre Biglossa e Lonchopria s.str.).

cingulata (Moure) comb.n. BRASIL, Paraná: Curitiba (localidade tipo).

Lonchopria (Ctenosibyne) cingulata Moure, 1956. Dusenia, Curitiba, 7 (6): 313-315, figs 4-6. Holótipo fêmea, alótipo e quatro parátipos no DZUP. - Michener, 1989. Univ. Kansas Sci. Bull. 53 (11): 681- 682.

Leioproctus (Ctenosibyne) cingulatus; Michener, 1965. Bull. Amer. Mus.

Nat. Hist. 130: 41. - McGinley, 1981. Univ. Calif. Publ. Ent. 91: 76.

Leioproctus (Lonchopria) cingulatus Toro \& de la Hoz, 1976. Rev. Soc.

Ent. Arg. 35 (1/4): 193-202.

Taxonomia. McGinley, 1981: 120-168 (análise fenética e cladística baseada em larvas).

Morfologia. Toro \& de la Hoz, 1976. Rev. Soc. Ent. Arg. 35(1/4): 193-202 (fatores mecânicos no isolamento reprodutivo, modificações das mandíbulas dos machos, do propódeo e do primeiro segmento metassomático das fềmeas). McGinley, 1981. Univ.Calif.Publ. Ent. 91: 76-77, figs 30-36 (morfologia da larva). 
Biologia. Michener \& Lange, 1957. Journ. Kansas Ent. Soc. 30 (2): 74-75, figs 1-3 (concentração de 2800 ninhos em barrancos no Barigui, Curitiba; descrição de ninhos; larvas e pupa). - Michener, Lange, Bigarella \& Salamuni, 1958, Dusenia, Curitiba, 8 (1): 4, 9, 17, 22; tabela I (ninhos agregados em barrancos).

\section{Edwyniana Moure, gen. rev.}

Spinolapis (Edwynia) Moure, 1951. Dusenia, Curitiba, 2 (3): 195-196.

Espécie-tipo: Pasiphae flavicornis Spinola, 1851. Designação original.

Edwyniana Moure, 1954. Dusenia, Curitiba, 5 (3/4): 165; nom. n. para Edwynia Moure (non Aldrich, 1930 [Diptera], Proc. ent. Soc. Washington 32: 26). - Michener, 1997. Sci. Papers, Nat. Hist.

Mus., Univ. Kansas 1: 21.

Leioproctus (Edwyniana); Michener, 1964. Systematic Zool. 13 (4): 187. - Michener, 1965: 41.

Leioproctus (Perditomorpha) (partim); Michener, 1989. Univ. Kansas Sci. Bull. 53 (11): 635.

flavicornis (Spinola) comb.n. CHILE, Santa Rosa (localidade tipo); Baños de Cauquenes; Coquimbo, Trancas, El Pangue, Quebrada-Seca, Chiches.

Pasiphae flavicornis Spinola, 1851, in Gay. Hist. Fis. Pol. Chile, Zool. 6: 228. Síntipos fêmea e macho no MIZT. - Reed, 1892. Acta Soc. Sci. Chili, Santiago, 2: 235.

Spinolapis (Edwynia) flavicornis; Moure, 1951. Dusenia, Curitiba, 2 (3): 195-196.

Leioproctus (Edwyniana) flavicornis; Michener, 1964. Systematic Zool. 13 (4): 187.

Leioproctus flavicornis; Toro, 1968. An. Mus. Hist. Nat., Valparaíso, 1: 125.

Leioproctus (Perditomorpha) flavicornis; Michener, 1989. Univ. Kansas Sci. Bull. 53 (11): 635, 636, 638.

Taxonomia. Toro, 1968. An. Mus. Hist. Nat., Valparaíso, 1: 125 (ao descrever duas espécies novas, no gênero Leioproctus, comenta que se assemelham externamente a L. flavicornis); 131(inclui na chave).

Biologia. Moure, 1951: 195 (distribuição geográfica e captura nos meses de setembro e outubro).

herrerae (Toro) comb.n. CHILE, Coquimbo: Los Fierros (localidade tipo); Valparaíso, Cuesta de Zapata.

Leioproctus herrerae Toro, 1968. An. Mus. Hist. Nat., Valparaíso, 1: 128-131, figs 4, 5 a-c, 6 e 7. Holótipo macho e alótipo na CTV; parátipo macho no UCVC e no MHNS.

Leioproctus (Perditomorpha) herrerae; Michener, 1989. Univ. Kansas Sci.

Bull. 53 (11): 635, 636, 638.

Taxonomia. Toro, 1968: 125(comenta que esta espécie poderia ser incluida em Edwyniana); 131(inclui na chave).

mourei (Toro) comb.n. CHILE, Valparaíso: Belloto (localidade tipo), Quilpué, El Salto, Peñuelas.

Leioproctus mourei Toro, 1968. An. Mus. Hist. Nat., Valparaíso, 1: 125- 
128, figs 1-3. Holótipo macho e alótipo na CTV; parátipos machos e fêmeas na coleção da UCVC.

Leioproctus (Perditomorpha) mourei; Michener, 1989. Univ. Kansas Sci. Bull. 53 (11): 635, 636, 638, 639.

Taxonomia. Toro, 1968: 125 (comenta que esta espécie poderia ser incluida em Edwyniana por assemelhar-se a L. flavicornis na aparência externa); 131 (chave). tristis (Spinola). CHILE (localidade tipo), Províncias centrais.

Pasiphae tristis Spinola, 1851, in Gay. Hist. Fis. Pol. Chile, Zool. 6: 230. Síntipo macho no MIZT.

Edwyniana tristis; Michener \& Lange, 1957. Journ. Kansas Ent. Soc. 30 (2): 72.

Leioproctus (Edwyniana) tristis; Michener, 1964. Systematic Zool. 13 (4): 187.

Leioproctus (Perditomorpha) tristis; Michener, 1989. Univ. Kansas Sci. Bull. 53 (11): 638.

Taxonomia. Spinola, 1851:230 (descreveu fêmea e macho, somente o macho foi localizado no MIZT). - Cockerell, 1905. Trans. Amer. Ent. Soc. 31: 317-318 (redescrição e comparação com outros gêneros). - Cockerell, 1917. Ann. Mag. Nat. Hist. (8) 20: 439 (comparou com P. leucostoma). - Cockerell, 1926. Ann. Mag. Nat. Hist. (9) 17: 220 (comparou com P. peruviana).

Biologia. Janvier, 1933. Ann. Sci. Nat., Zool., Paris, (10) 16: 327-330 (descrição do ninho e da larva, visita a flores de Eryngium paniculatum, Senecio hualtata, Solanum, Fuchsia macrostemma, Escallonia rubra e Loasa).

\section{Ethalonchopria Michener, stat. nov.}

Eulonchopria (Ethalonchopria) Michener, 1989. Univ. Kansas Sci. Bull. 53 (11): 670-671. - Michener, 1997. Sci. Papers, Nat. Hist. Mus., Univ. Kansas 1: 23.

Espécie-tipo: Apista gaullei Vachal, 1909. Designação original.

Taxonomia: Michener, 1985. Journ. Kansas Ent. Soc. 58 (2): 236-239; fig. 1 (colocou Apista limbella como provável sinônimo de Apista gaullei; e relacionou na chave). - Michener, 1989: 670 (comenta que este sub-gênero é menos estranho que Eulonchopria s.str., é mais como os outros Paracolletini).

gaullei (Vachal) comb.n. BolívIA, Mapiri (localidade tipo). BRASIL, Santa Catarina.

Apista gaullei Vachal, 1909. Rev. d'Ent., Caen, 28: 37. Holótipo fêmea no MNHP.

Eulonchopria gaullei; Michener, 1985. Journ. Kansas Ent. Soc. 58 (2): 237.

Eulonchopria (Ethalonchopria) gaullei; Michener, 1989. Univ. Kansas Sci.

Bull. 53 (11): 670.

Taxonomia. Michener, 1985: 237-238 (propõe nova combinação, coloca $A$. limbella como provavel sinônimo, compara com L. psaenythioides e inclui na chave). 
limbella (Vachal) comb.n. BoLíviA, Mapiri (localidade tipo).

Apista limbella Vachal, 1909. Rev. d'Ent., Caen, 28: 37. Holótipo fêmea no MNHP.

Eulonchopria (Ethalonchopria) limbella; Michener, 1989. Univ. Kansas Sci. Bull. 53 (11): 670.

Taxonomia. Michener, 1985. Journ. Kansas Ent. Soc. 58 (2): 237-238 (notas, chave). - Michener, 1989: 670-671 (limbella e gaullei podem ser a mesma espécie).

\section{Eulonchopria Brèthes}

Eulonchopria Brèthes, 1909. An. Mus. Nac., Buenos Aires, 19: 247. - Michener, 1964. Systematic Zool. 13 (4): 190. - Michener, 1965. Bull. Amer. Mus. Nat. Hist. 130: 40. - Michener, 1997. Sci. Papers, Nat. Hist. Mus., Univ. Kansas 1: 23.

Espécie-tipo: Eulonchopria psaenythioides Brèthes, 1909. Monotipia.

Taxonomia. Michener, 1963. Ann. Ent. Soc. America 56: 844-849; figs 1-15 (redescrição do gênero e das espécies; chave). - Michener, 1985. Journ. Kansas Ent. Soc. 58 (2): 236-239 (notas, chave). - Michener, 1989: 669-671; fig. $22 \mathrm{j}$ (redescrição do gênero e chave para os sub-gêneros).

flavescens Friese comb.n. CosTA RICA, San José (localidade tipo).

Parapsaenythia flavescens Friese, 1916. Stett. Ent. Ztg. 77: 303 (partim). Síntipos no MNHU.

Taxonomia. Friese, 1916: 303 (relacionou fêmeas de San José, Costa Rica e de Villa Rica, Paraguai). - Moure identificou, no MNHU, como Eulonchopria flavescens os exemplares de San José, Costa Rica, coletados por Schmidt.

oaxacana Michener. MÉXICO, Oaxaca, 20 milhas a leste de El Camarón (localidade tipo).

Eulonchopria oaxacana Michener, 1963. Ann. Ent. Soc. Amer. 56: 845, 848-849, figs 3, 4, 10-12. Holótipo fềmea e alótipo no CAS; parátipos no SEMK.

Eulonchopria (Eulonchopria) oaxacana; Michener, 1989. Univ. Kansas Sci. Bull. 53 (11): 671.

Taxonomia. Ayala, 1988. Folia Entomol. Mex. 77: 452 (chave). - Michener, 1989: 671 (cita).

Biologia. Michener, 1963: 849 (R. W. Thorp identificou pólen de Acacia ou Inga nas fêmeas).

psaenythioides Brèthes. PARAGUAI, San Bernardino (localidade tipo); Villa Rica. Argentina, Salta; Tucumán. Brasil, Minas Gerais; Rio de Janeiro; São Paulo; Paraná; Santa Catarina.

Eulonchopria psaenythioides Brèthes, 1909. An. Mus. Nac., Buenos Aires, 19: 248-249. Síntipos fêmea e macho no MBR.

Psaenythia tomentifera Strand, 1910. Zool. Jahrb., Abt. Syst. 29: 498. Síntipos no MNHU. Syn. $\mathbf{n}$.

Psaenythia bernardinensis Strand, 1910. Zool. Jahrb., Abt. Syst. 29: 499. Holótipo no MNHU. Syn. $\mathbf{n}$. 
Parapsaenythia flavescens Friese, 1916. Stett. Ent. Ztg. 77: 303 (partim). Síntipos no MNHU. Syn. n.

Eulonchopria (Eulonchopria) psaenythioides; Michener, 1989. Univ. Kansas Sci. Bull. 53 (11): 671.

Taxonomia. Ducke, 1912: 76 (nota). - Schrottky, 1913. An. Soc. Ci. Arg. 75: 246. - Friese, 1916: 303 (ao descrever P. flavescens Friese comparou com Parapsaenythia argentina Friese, 1908). - Michener, 1963. Ann. Ent. Soc. America 56: 845-847, figs 5, 6, 13-15 (redescrição; distribuição geográfica). - Michener, 1989: 671. - Moure identificou, no MNHU, como E. psaenythioides os exemplares de $P$. flavescens de Villa Rica, Paraguai, coletados por Burgdorf e relacionados por Friese, 1916: 303. soidea)

Biologia. Cure et al., 1993. Revista Ceres 40 (228): 138, 147 (em Mimo-

punctatissima Michener. Estados Unidos, Arizona: Santa Cruz County, 5 milhas a oeste de Patagonia (localidade tipo); MÉXICO, Guerrero.

Eulonchopria punctatissima Michener, 1963. Ann. Ent. Soc. Amer. 56: 845 , 847-848, figs 1, 2, 7-9. Holótipo fêmea, alótipo e parátipos no SEMK; parátipos na UAT.

Eulonchopria (Eulonchopria) punctatissima; Michener, 1989. Univ. Kansas Sci. Bull. 53 (11): 671.

Taxonomia. Ayala, 1988. Folia Entomol. Mex. 77: 452 (chave).-Michener, 1989: 668 (fig. 22 j), 671 (nota).

Biologia. Michener, 1963: 848 (em flores de "Acacia augustis", presumivelmente $A$. angustissima; R. W. Thorp determinou pólen de uma Composta, talvez Baccharis na escopa).

\section{Glossopasiphae Michener, stat. nov.}

Leioproctus (Glossopasiphae) Michener, 1989. Univ. Kansas Sci. Bull. 53 (11): 643. - Michener, 1997. Sci. Papers, Nat. Hist. Mus., Univ. Kansas 1: 26.

Espécie-tipo: Leioproctus (Glossopasiphae) plaumanni Michener. Designação original.

plaumanni (Michener) comb.n. BRASIL, Santa Catarina: Seara (Nova Teutônia) (localidade tipo).

Leioproctus (Glossopasiphae) plaumanni Michener, 1989. Univ. Kansas Sci. Bull. 53 (11): 643, 692, figs 6 a-k; 19 d. Holótipo macho e alótipo no SEMK.

Taxonomia. Michener, 1989: 692 (considerou a forma e a coloração como de Andrena carlini Cockerell, e superficialmente similar a L. fulvoniger da mesma localidade de L. plaumanni).

\section{Halictanthrena Ducke}

Halictanthrena Ducke, 1907. Ztschr. Hym. Dipt. 7: 364. - Michener, 1997. Sci. Papers, Nat. Hist. Mus., Univ. Kansas 1: 27.

Espécie-tipo: Halictanthrena malpighiacearum Ducke. Monotipia. 
Leioproctus (Halictanthrena); Michener, 1964. Systematic Zool. 13 (4): 187. - Michener, 1965. Bull. Amer. Mus. Nat. Hist. 130: 42. - Michener, 1989. Univ. Kansas Sci. Bull. 53 (11): 649-651, figs $9 \mathrm{a}-\mathrm{f}, 10 \mathrm{i}$.

malpighiacearum (Ducke). BRASIL, Minas Gerais: Barbacena (localidade tipo).

Halictanthrena malpighiacearum Ducke, 1907. Ztschr. Hym. Dipt. 7: 364-365. Lectótipo e lectoalótipo no MPEG.

Leioproctus (Halictanthrena) malpighiacearum; Michener, 1989. Univ.

Kansas Sci. Bull. 53 (11): 649-651; figs 9 a-f, 10 i.

Taxonomia. Nascimento, 1979. Bol. Mus. Paraense Emílio Goeldi, Zoologia 98: 6 (designação de lectótipo e lectoalótipo por indicação de Moure \& Michener). - Michener, 1989: 651 (citou dois síntipos no MNHU, designou a fêmea como lectótipo). - Obrecht \& Huber, 1993. Jahrb. Naturhist. Mus. Bern 11: 168 (informações sobre os tipos).

Biologia. Ducke, 1907: 365 (em flores de Malpighiaceae, em outubro e novembro, em campos).

\section{Hexantheda Ogloblin}

Hexantheda Ogloblin, 1948. Notas Mus. La Plata, Zool. 13 (106): 172. - Michener, 1964. Systematic Zool. 13 (4): 190. - Michener, 1965. Bull. Amer. Mus. Nat. Hist. 130: 40. - Michener, 1997. Sci.

Papers, Nat. Hist. Mus., Univ. Kansas 1: 29.

Espécie-tipo: Hexantheda missionica Ogloblin. Designação original.

Leioproctus (Hexantheda); Michener, 1989. Univ. Kansas Sci. Bull. 53 (11): 652-653.

Taxonomia. Michener, 1989: 653 (considerou como sub-gênero de Leioproctus, provavelmente derivado de um ancestral de Perditomorpha).

missionica Ogloblin. ARGENTINA, Misiones: Loreto (localidade tipo) e San Ignacio; Formosa. BRASIL, Paraná.

Hexantheda missionica Ogloblin, 1948. Notas Mus. La Plata, Zool, 13 (106): 173-177, figs 16 -23. Holótipo, alótipo e parátipos no MLP.

Leioproctus (Hexantheda) missionica; Michener, 1989. Univ. Kansas Sci. Bull. 53 (11): 652-653.

Taxonomia. Michener, 1989: 652-653, figs 11 a-f, 16 b. (redescrição).

\section{Holmbergeria Joergensen}

Holmbergeria Joergensen, 1912. Zool. Jahrb., Abt. Syst. 32: 100. - Michener, 1997. Sci. Papers, Nat.

Hist. Mus., Univ. Kansas 1: 29.

Espécie-tipo: Holmbergeria cristariae Joergensen. Monotipia.

Leioproctus (Perditomorpha); Michener, 1965. Bull. Amer. Mus. Nat. Hist. 130: 41(= Holmbergia [sic])

Leioproctus (Holmbergeria); Michener, 1989. Univ. Kansas Sci. Bull. 53 (11): 646.

Taxonomia. Michener, 1965: 41 (considerou Holmbergia [sic] na sinonímia do sub-gênero Perditomorpha). - Michener, 1989: 646 (redescrição incluindo duas espécies bastante similares: $L$. cristariae com as pernas e o metasoma pretos e $L$. rubriventris com as pernas e os dois ou três tergos basais avermelhados). 
cristariae Joergensen. ARgentina, Mendoza, Chacras de Coria (localidade tipo) e San Ignacio.

Holmbergeria cristariae Joergensen, 1912. Zool. Jahrb., Abt. Syst. 32: 100. Lectótipo macho designado por Michener, 1989. Univ. Kansas Sci.

Bull. 53 (11): 693, no MLP.

Leioproctus (Perditomorpha) cristariae; Michener, 1965. Bull. Amer. Mus.

Nat. Hist. 130: 41.

Leioproctus (Holmbergeria) cristariae; Michener, 1989. Univ. Kansas Sci.

Bull. 53 (11): 646.

Taxonomia. Michener, 1965: 41 (citou a espécie).

Biologia. Joergensen, 1912: 101 (comuns de outubro a dezembro em Larrea divaricata e Cristaria loasifolia).

rubriventris (Friese) comb.n. ARGENTINA, Mendoza: Chacras de Coria (localidade tipo). PARAgUAI, S. Bernardino.

Biglossa rubriventris Friese, 1909, in Strand. Deutsch. Ent. Ztschr. : 235. Síntipos fêmea e macho no MNHU.

Leioproctus (Holmbergeria) rubriventris; Michener, 1989. Univ. Kansas

Sci. Bull. 53 (11): 646; figs 7 i-o.

Taxonomia. Strand, 1910. Zool. Jahrb., Abt. Syst. 29: 456 (nota). - Ducke, 1912. Zool. Jahrb., Abt. Syst. 34: 80 (nota).

Biologia. Friese, 1909 (fêmea em Cristaria latifolia, Malvaceae).

\section{Hoplocolletes Michener, stat. nov.}

Leioproctus (Hoplocolletes) Michener, 1965. Bull. Amer. Mus. Nat. Hist. 130: 42. - Michener, 1989. Univ. Kansas Sci. Bull. 53 (11): 646. - Michener, 1997. Sci. Papers, Nat. Hist. Mus., Univ. Kansas 1: 29.

Espécie-tipo: Dasycolletes ventralis Friese. Designação original.

Taxonomia. Michener, 1989: 646-647 (redescrição do sub-gênero e nota comparativa).

ventralis (Friese) comb.n. BRASIL (localidade tipo).

Dasycolletes ventralis Friese, 1924. Konowia, Wien, 3: 218. Holótipo fềmea no AMNH.

Paracolletes ventralis; Cockerell, 1929. Amer. Mus. Novitates 343: 3-4.

Leioproctus (Hoplocolletes) ventralis; Michener, 1989. Univ. Kansas Sci. Bull. 53 (11): 646-647.

Taxonomia. Friese, 1924:218. (descreve $D$. ventralis de Sydney [Austrália]). - Cockerell, 1929: 3-4 (redescreve a fêmea num outro gênero, que engloba espécies australianas). - Michener, 1965: 41-42 (compara o holótipo de Sidney, do AMNH, com uma fêmea do Rio de Janeiro, Brasil e conclui que Sidney é um erro de etiqueta, sendo Brasil a localidade válida).

Morfologia. - Michener, 1989: 645 (figs p, q). 


\section{Kylopasiphae Michener, stat. nov.}

Leioproctus (Kylopasiphae) Michener, 1989. Univ. Kansas Sci. Bull. 53 (11): 641. - Michener, 1997.

Sci. Papers, Nat. Hist. Mus., Univ. Kansas 1: 31.

Espécie-tipo: Leioproctus (Kylopasiphae) pruinosus Michener. Designação original.

pruinosa (Michener) comb.n. ARgENTINA, Tucumán: $5 \mathrm{~km}$ a leste de Amaicha (localidade tipo); Catamarca; La Rioja; Neuquén.

Leioproctus (Kylopasiphae) pruinosus Michener 1989. Univ. Kansas Sci. Bull. 53 (11): 641, 690-691; figs 5 a-f; 10 i; 23 e. Holótipo macho, alótipo fêmea e parátipos no SEMK; parátipos no DZUP, MBR e outras coleções conforme Michener, 1989: 691.

Taxonomia: Michener, 1989: 641, 690 (lembra Lonchopria robertsi e Leioproctus (Pygopasiphae) mourellus, que ocorrem na mesma área).

Biologia: Michener, 1989: 691 (em Catamarca coletada em Larrea divaricata, Larrea cuneifolia, Bulnesia retama, sem pólen na escopa).

\section{Lonchoprella Michener, stat. nov.}

Lonchopria (Lonchoprella) Michener, 1989. Univ. Kansas Sci. Bull. 53 (11): 673. - Michener, 1997.

Sci. Papers, Nat. Hist. Mus., Univ. Kansas 1: 33.

Espécie-tipo: Lonchopria (Lonchoprella) annectens Michener. Designação original.

Taxonomia. Michener, 1989: 673 (este sub-gênero difere dos outros sub-gêneros de Lonchopria por ter a escopa tibial similar à de Leioproctus).

annectens (Michener) comb.n. ARGENTINA, Santiago del Estero, Matará (localidade tipo), Las Termas; Catamarca, Colpes, Andalgalá.

Lonchopria (Lonchoprella) annectens Michener, 1989. Univ. Kansas Sci. Bull. 53 (11): 673, 699-700; figs 24 a- k, 31 e. Holótipo macho e alótipo no SEMK; parátipo no MBR e outras coleções conforme Michener, 1989: 700 .

Biologia. Michener, 1989: (machos em Prosopis alba, em Las Termas e Andalgalá, e um macho em Zucagnia em Colpes).

\section{Lonchopria Vachal}

Lonchopria Vachal, 1905. Bull. Soc. Ent. Fr. : 204. - Michener, 1997. Sci. Papers, Nat. Hist. Mus., Univ. Kansas 1: 33.

Espécie-tipo: Lonchopria herbsti Vachal = Colletes zonalis Reed, 1892. Monotipia .

Leioproctus (Lonchopria); Michener, 1964. Systematic Zool. 13 (4): 187. - Michener, 1965. Bull. Amer. Mus. Nat. Hist. 130: 41.

Lonchopria (Lonchopria); Michener, 1989. Univ. Kansas Sci. Bull. 53 (11): 680-681.

Taxonomia. Michener, 1989: 681 (compara com Biglossa, Ctenosibyne e Porterapis)

luteipes (Friese). CHILE, Rancágua (localidade tipo).

Caupolicana (Biglossa) luteipes Friese, 1916. Stett. Ent. Ztg. 77: 163. Holótipo fêmea no MNHU.

Leioproctus (Lonchopria) luteipes; Michener, 1964. Systematic Zool. 13 
(4): 187. - Michener, 1965. Bull. Amer. Mus. Nat. Hist. 130: 41. - Toro, 1973. Rev. Chilena Ent. 7: 156.

Lonchopria (Lonchopria) luteipes; Michener, 1989. Univ. Kansas Sci. Bull. 53 (11): 681.

Taxonomia. Toro, 1973: 156 (traduz a descrição).

rufitorax Ruiz. CHILE, Termas de Manzanares a leste de Cura-Cautín (localidade tipo); Pucón; Concepción; Hacienda las Mercedes.

Lonchopria marginata rufitorax Ruiz, 1944. Rev. Chilena Hist. Nat. 47: 228. Síntipos fêmeas e machos no MHPN.

Leioproctus (Lonchopria) rufithorax [sic]; Toro, 1973. Rev. Chilena Ent. 7: 146, 154- 156, 171, 172; figs 106-109.

Lonchopria (Lonchopria) rufithorax [sic]; Michener, 1989. Univ. Kansas Sci. Bull. 53 (11): 681.

Taxonomia. Toro, 1973: 146, 154-156 (chave; redescrição).

Biologia. Ruiz, 1944: 228 (ninhos nas Termas de Manzanares).

similis (Friese). CHILE, Concepción (localidade tipo); Coquimbo, Peñuelas, Guayacán, Choros; Valparaíso, Lilenes, Quinteros, Horcones, Concón, Montagua; Maule, Cauquenes; Arauco.

Biglossa similis Friese, 1906. Ztschr. Hym. Dipt. 6: 377-378. Síntipos fêmea e macho no MNHU.

Lonchopria similis; Ducke, 1912. Zool. Jahrb., Abt. Syst. 34: 80.

Leioproctus (Lonchopria) similis; Michener, 1965. Bull. Amer. Mus. Nat. Hist. 130: 41.

Lonchopria (Lonchopria) similis; Michener, 1989. Univ. Kansas Sci. Bull. 53 (11): 681; fig. $30 \mathrm{~h}$.

Taxonomia. Friese, 1908. Flora og Fauna 10: 13, 14, 16 (chave; cópia da descrição). - Toro, 1973. Rev. Chilena Ent. 7: 146, 148-151, 171-172; figs 88-96. (chave; redescrição; distribuição geográfica; morfometria).

Biologia. Ruiz, 1944. Rev. Chilena Hist. Nat. 47: 230-231 (15 a 20 machos, à noite, em flores de Cereus litoralis). - Toro \& de la Hoz, 1976. Rev. Soc. Ent. Arg. 35 (1/4): 193-202 (fatores mecânicos no isolamento reprodutivo, modificações das mandíbulas dos machos, do propódeo e do primeiro segmento metassomático das fêmeas).

zonalis (Reed, 1892). CHILE, Coquimbo (localidade tipo); Valparaiso; Concepción, Concon, Cura-Cautín (Termas de Manzanares).

Colletes marginata Spinola, 1851, in Gay. Hist. Fis. Pol. Chile, Zool. 6: 223 (nom. praeoc.). Síntipos fêmea e machos no MIZT, examinados por J. S. Moure.

Colletes zonalis Reed, 1892. Act. Soc. Sci. Chili 2: 234. (nom. n. para Colletes marginata Spinola, 1851, non Colletes marginata Smith, 1846, uma espécie européia).

Colletes spinolae Dalla Torre, 1896. Cat. Hym. 10: 44. (nom. n. para C. marginata Spinola, 1851). 
Lonchopria herbsti Vachal, 1905. Bull. Soc. Ent. Fr. : 204. Síntipo fêmea no MNHP, examinado por J. S. Moure.

Biglossa marginata; Friese, 1906. Zeitschr. Hym. Dipt. 6: 374, 375, 377.

Lonchopria marginata; Alfken, 1907. Zeitschr. Hym. Dipt. 7: 79.

Lonchopria zonalis; Cockerell, 1917. Ann. Mag. Nat. Hist. (8) 9: 480. -

Moure, 1948. Rev. Ent., Rio de Janeiro, 19 (1/2): 315.

Leioproctus (Lonchopria) zonalis; Michener, 1964. Systematic Zool. 13

(4): 187. - Michener, 1965. Bull. Amer. Mus. Nat. Hist. 130: 41. - Toro,

1973, Rev. Chilena Ent. 7: 145, 146, 151-154; 171, 172; figs 97-105. -

McGinley, 1981. Univ. Calif. Publ. Ent. 91: 73.

Lonchopria (Lonchopria) zonalis; Michener, 1989. Univ. Kansas Sci. Bull.

53 (11): 680, 681; fig. $31 \mathrm{~h}$.

Taxonomia. Vachal, 1905: 204 (descreveu fêmea e macho de L. herbsti). Friese, 1906: 377 (redescrição). - Vachal, 1909. Rev. d'Ent., Caen, 28: 37(L. herbsti não é realmente $C$. marginata Spinola). - Ducke, 1912: 79 (L. marginata $=$ C. spinolae $=$ L. herbsti). - Ruiz, 1944. Rev. Chilena Hist. Nat. 47: 226-228 (redescrição). - Michener, 1965: 41 (coloca em sinonímia zonalis = spinolae = marginata). - Toro, 1973: 151 (sinonímia $z$ onalis $=$ marginata $=$ spinolae $=$ herbst $i)$. - Nascimento, 1979. Bol. Mus. Paraense Emílio Goeldi, Zoologia 98: 6 (citou Lonchopria marginata Friese [sic] [o autor é Spinola]- McGinley, 1981: 120-168 (análise fenética e cladística, com base na larva). - Michener, 1989: 680 (Lonchopria herbsti $=$ Colletes $z$ nalis Reed, 1892).

Morfologia. McGinley, 1981. Univ. Calif. Publ. Ent. 91: 73-76, figs 23-29 (descrição da larva).

Biologia. Claude-Joseph, 1926. Ann. Sci. Nat. Zool., Paris, 10: 140-142 (em flores de Quillaja saponaria; descrição de ninhos; coleta de pólen; morfologia das larvas). - Gazulla \& Ruiz, 1928. Rev. Chilena Hist. Nat. 30: 301 (em flores de "Quillay"). - Janvier, 1933. Ann. Sci. Nat. Zool., Paris, (10) 16: 325-326 (parasitada por Isepeolus luctuosus Spinola, Isepeolus viperinus Friese e Sphecodes friesei Herbst). - Ruiz, 1944. Rev. Chilena His. Nat. : 222, 226, 227 (em flores de "quillay", Loasa; descrição de ninhos em Termas de Manzanares). - Toro \& de la Hoz, 1976. Rev. Soc. Ent. Arg. 35 (1/4): 193-202 (fatores mecânicos no isolamento reprodutivo, modificações das mandíbulas dos machos, do propódeo e do primeiro segmento metassomático das fêmeas).

\section{Lonchorhyncha Michener}

Lonchorhyncha Michener, 1989. Univ. Kansas Sci. Bull. 53 (11): 667-668. - Michener, 1997. Sci. Papers, Nat. Hist. Mus., Univ. Kansas 1: 33.

Espécie-tipo: Diphaglossa ecuadoria Friese. Designação original.

ecuadoria (Friese). EQUADOR, Guayaquil (localidade tipo), Azuay, Saraguro, $2900 \mathrm{~m}$.

Diphaglossa ecuadoria Friese, 1925. Stett. Ent. Ztg. 86: 10-11. Holótipo fêmea no MNHU.

Lonchorhyncha ecuadoria; Michener, 1989. Univ. Kansas Sci. Bull. 53

(11): 667-669. 
Taxonomia. Michener, 1989: 629 (taxonomia.), 631 (chave), 667-669 (descrição do gênero), 698-699 (redescrição da fêmea, descrição do macho, taxonomia), figs $22 \mathrm{a}-\mathrm{i}, 23 \mathrm{a}-\mathrm{d}, 31 \mathrm{c}$.

Biologia. Friese, 1925: 11 (fềmea coletada em abril em Cucurbita).

\section{Niltonia Moure}

Niltonia Moure, 1964. Bol. Univ. Paraná, Zool., Curitiba, II (4): 52-55. -Michener, 1989. Univ. Kansas

Sci. Bull. 53 (11): 665. - Michener, 1997. Sci. Papers, Nat. Hist. Mus., Univ. Kansas 1: 41. Espécie-tipo Niltonia virgilii Moure. Designação original.

virgilii Moure. BRASIL, Rio de Janeiro; São Paulo: Pariquera-Açu (localidade tipo); Paraná: Paranaguá, Alexandra; Santa Catarina.

Niltonia virgilii Moure, 1964. Bol. Univ. Paraná, Zool., Curitiba, II (4):

55-56; fig. 1. Holótipo fêmea no MZSP e parátipo fêmea no DZUP.

Taxonomia. Laroca \& Almeida, 1985. Revta bras. Ent. 29 (2): 290-291 (descrição do macho; figs 1-3). - Michener, 1989: 665-667 (redescrição do gênero; figs 21 a-n, 31 b; compara com Brachyglossula).

Morfologia. Laroca; Michener \& Hofmeister, 1989. Journ. Kansas Ent. Soc. 62 (3): 400, 403- 409; figs 1 - 10 (detalhes da estrutura da glossa e dos palpos labiais).

Biologia. Laroca \& Almeida, 1985. Revta bras. Ent. 29 (2): 289-297, fig. 4 (visita flores e coleta pólen de Jacaranda puberula, Bignoniaceae, de agosto a outubro; alimentação em laboratório; experimento com flor de Paulownia fortunei; comportamento alimentar).

\section{Nomiocolletes Brèthes}

Nomiocolletes Brèthes, 1909. An. Mus. Nac., Buenos Aires, 19: 455. - Michener, 1997. Sci. Papers, Nat. Hist. Mus., Univ. Kansas 1: 41.

Espécie-tipo: Nomia joergenseni Friese. Designação original.

Leioproctus (Nomiocolletes); Michener, 1964. Systematic Zool. 13 (4): 187. - Michener, 1965. Bull.

Amer. Mus. Nat. Hist. 130: 41. - Michener, 1989. Univ. Kansas Sei. Bull. 53 (11): 660.

Taxonomia. Moure, 1948. Rev. Ent., Rio de Janeiro, 19 (1/2): 313 (considerou um bom gênero, distinto de Lonchopria e relacionou os caracteres de Nomiocolletes). - Michener, 1989: 660-663. figs 16 h, 17 a-g, 18 a-f (nota comparativa).

arnaui Moure. ARgENTINA, Tucumán: San Pedro de Colalao, Tacanas (localidade tipo).

Nomiocolletes arnaui Moure, 1949. Rev. Ent., Rio de Janeiro, 20 (1/3): 441-442. Holótipo fêmea, alótipo macho e dois parátipos no IML; sete parátipos no DZUP.

Leioproctus (Nomiocolletes) arnaui; Michener, 1964. Systematic Zool. 13

(4): 187. - Michener, 1965. Bull. Amer. Mus. Nat. Hist. 130: 41. Michener, 1989. Univ. Kansas Sci. Bull. 53 (11): 662.

Biologia. Schlindwein, 1995. Wildbienen und ihre Trachtpflanzen..., 
Stuttgart: 89 (em Eryngium horridum, E. nudicaule, Hypochoeris pampasica e Croton thermarum).

cearensis (Ducke). BRASIL, Ceará: Baturité (localidade tipo), Caridade.

Nomia cearensis Ducke, 1908. Rev. d'Ent., Caen, 27: 64-65. Material tipo não examinado.

Lonchopria cearensis; Ducke, 1910. Rev. Trim. Inst. Ceará 24: 44.

Nomiocolletes cearensis; Moure, 1949. Rev. Ent., Rio de Janeiro, 20 (1/3): 442.

Leioproctus (Nomiocolletes) cearensis; Michener, 1964. Systematic Zool. 13 (4): 187. - Michener, 1965. Bull. Amer. Mus. Nat. Hist. 130: 41. Michener, 1989. Univ. Kansas Sci. Bull. 53 (11): 662.

Taxonomia. Ducke, 1908: 64-65 (comparou com $N$. jenseni e $N$. joergenseni). - Ducke, 1910. Deutsch. Ent. Ztschr. : 368 (nota descritiva). - Ducke, 1910, Rev. d'Ent., Caen 28: 80 (como Lonchopria, distribuição geográfica). - Moure, 1949: 442 (nota comparativa).

Biologia. Ducke, 1910. Rev. d'Ent., Caen, 28: 80 (machos e fêmeas de abril ao início de maio).

jenseni (Friese). Argentina, Mendoza (localidade tipo), Pedregal, Chacras de Cória, Estação La Paz; Santa Rosa; Córdoba; Tucumán, Tacanas; Neuquén.

Nomia jenseni Friese, 1906. Flora og Fauna 8: 90. Material tipo não examinado.

Lonchopria jenseni; Vachal, 1909. Rev. d'Ent., Caen, 28: 37.

Nomiocolletes jenseni; Brèthes, 1909. An. Mus. Nac., Buenos Aires, 17: 456. - Moure, 1948. Rev. Ent., Rio de Janeiro, 19 (1/2): 313. - Moure, 1949. Rev. Ent., Rio de Janeiro, 20 (1/3): 442.

Leioproctus (Nomiocolletes) jenseni; Michener, 1964. Systematic Zool. 13 (4): 187. - Michener, 1965. Bull. Amer. Mus. Nat. Hist. 130: 41. Michener, 1989. Univ. Kansas Sci. Bull. 53 (11): 662.

Taxonomia. Ducke, 1908. Rev. d'Ent., Caen, 27: 64-65 (nota comparativa). - Friese, 1910. Zool. Jahrb., Abt. Syst. 29: 643 (distribuição geográfica).

Biologia. Friese, 1906: 90 (fêmea e macho coletados em dezembro). Joergensen, 1909. Deutsch. Ent. Ztschr. : 223 (de novembro a março em Prosopis, Acacia e Baccharis salicifolia). - Joergensen, 1912. An. Mus. Nac., Buenos Aires, 22: 303 (raro em Pedregal e Chacras de Cória, abundante em La Paz, sobre Heterothalamus spartioides, mais raro sobre Ximenedia microptera, Baccharis salicifolia, Prosopis alpataco, P. campestris, Acacia furcata).

joergenseni (Friese). ArgentinA, Mendoza (localidade tipo), Punta del Agua, Potrerillos, Pedregal, Puente del Inca, 2710m; Catamarca.

Nomia joergenseni Friese, 1908. Flora og Fauna 10: 26. Síntipo macho no MNHU.

Lonchopria joergenseni; Vachal, 1909. Rev. d'Ent., Caen, 28: 37.

Nomiocolletes joergenseni; Brèthes, 1909. An. Mus. Nac., Buenos Aires, 17: 455. - Moure, 1949. Rev. Ent., Rio de Janeiro, 20 (1/3): 442. 
Leioproctus (Nomiocolletes) joergenseni; Michener, 1964. Systematic Zool. 13 (4): 187. - Michener, 1965. Bull. Amer. Mus. Nat. Hist. 130: 41. - Michener, 1989. Univ. Kansas Sci. Bull. 53 (11): 662; figs 16 h, $17 \mathrm{a}-\mathrm{g}$.

Taxonomia. Friese, 1908: 26 (descreveu macho e fêmea)-Ducke, 1908, Rev. d'Ent., Caen, 27: 64-65 (nota comparativa). - Joergensen, 1912. Zool. Jahrb., Abt. Syst. 32: 106 (chave e distribuição geográfica).

Biologia. Jensen-Haarup, 1908. Flora og Fauna 10: 100-101 (em flores de Baccharis marginalis var. coerulescens; ninhos no solo). - Joergensen, 1909. Deutsch. Ent. Ztschr. : 58 (novembro a maio, machos e fêmeas em Grindelia pulchella, Senecio pinnatus, Tessaria absinthioides, Baccharis salicifolia, B. subulata, Cyclolepis genistoides, Telesperma scabriosoides, Heterothalamus spartioides, Prosopis alpataco, P. campestris, Acacia furcata, Abutilon mendocina [sic], Larrea divaricata, machos em Phoeniculum piperitum. - Joergensen, 1912. An. Mus. Nac., Buenos Aires, 22: 303 (flores de Baccharis effusa e as citadas acima). - Schrottky, 1913. An. Soc. Ci. Arg. 75: 237 (distribuição geográfica).

simplicicrus (Michener) comb.n. PERU, Loreto, Pucallpa, 200m (localidade tipo).

Leioproctus (Nomiocolletes) simplicicrus Michener, 1989. Univ. Kansas Sci. Bull. 53 (11): 697-698. Holótipo macho no SEMK.

Taxonomia. Michener, 1989: 661-663, 697-698; figs $18 \mathrm{a}-\mathrm{f}$ (nota comparativa).

\section{Perditomorpha Ashmead}

Perditomorpha Ashmead, 1899. Trans. Amer. Ent. Soc. 26: 86. - Michener, 1997. Sci. Papers, Nat. Hist. Mus., Univ. Kansas 1: 47.

Espécie-tipo: Perditomorpha brunerii Ashmead. Monotipia.

Leioproctus (Perditomorpha); Michener, 1964. Systematic Zool. 13 (4): 187. - Michener, 1965. Bull. Amer. Mus. Nat. Hist. 130: 41. - Michener, 1989. Univ. Kansas Sci. Bull. 53 (11): 635.

arnaui Moure. ARgentina, Tucumán, San Pedro Colalao (localidade tipo).

Perditomorpha arnaui Moure, 1954. Dusenia, Curitiba, 5 (3/4): 168. Síntipos fêmea e macho no DZUP e IML.

Leioproctus (Bicolletes) arnaui; Michener, 1964. Systematic Zool. 13 (4): 187. - Michener, 1965. Bull. Amer. Mus. Nat. Hist. 130: 41.

Leioproctus (Perditomorpha) arnauellus Michener, 1989. Univ. Kansas

Sci. Bull. 53 (11): 688 (nom. n. para Perditomorpha arnaui Moure, 1954, pela homonímia com Leioproctus (Nomiocolletes) arnaui (Moure, 1949).

Taxonomia. Moure, 1954: 169 (comparou com P. brunerii). - Michener, 1964: 187. (nom. praeoc. em Leioproctus por Nomiocolletes arnaui Moure, 1949). - Michener, 1965: 41 (homônimo de L. (Nomiocolletes) arnaui (Moure, 1949). Michener, 1989: 635, 636. 638, 639 e 688 (citações como L. arnauellus). O nome arnauellus não foi acatado porque Perditomorpha e Nomiocolletes são gêneros válidos. 
brunerii Ashmead. Argentina, Santa Fé: Carcarañá (localidade tipo); BrASIL, Minas Gerais; São Paulo: Rio Claro, Guarulhos; Paraná: Curitiba, S. José dos Pinhais.

Perditomorpha brunerii Ashmead, 1899. Trans. Amer. Ent. Soc., 26: 86. Holótipo fêmea no USNM.

Leioproctus (Perditomorpha) brunerii; Michener, 1964. Systematic Zool. 13 (4): 187. - Michener, 1965. Bull. Amer. Mus. Nat. Hist. 130: 41. Michener, 1989. Univ. Kansas Sci. Bull. 53 (11): 635-639; figs $3 \mathrm{~g}-\mathrm{j}$.

Taxonomia. Moure, 1954. Dusenia, Curitiba, 5 (3/4): 169 (compara com $P$. arnaui; distribuição geográfica).

Biologia. Michener \& Lange, 1957. Journ. Kansas Ent. Soc. 30 (2): 77 (nidificação). - Michener; Lange; Bigarella \& Salamuni, 1958. Dusenia, Curitiba, 8 (1): 4 (ninhos de abelhas em barrancos). - Schlindwein, 1995. Wildbienen und ihre Trachtpflanzen..., Stuttgart: 89 (em flores de Abutilon pauciflorum, Malvaceae).

inconspicua (Michener) comb.n. ARGENTINA, Salta: Tartagal, $502 \mathrm{~m}$ (localidade tipo), Campos Durán; Bolívia, Santa Cruz de La Sierra, a oeste de Montero; San Javier, $600 \mathrm{~m}$.

Leioproctus (Perditomorpha) inconspicuus Michener, 1989. Univ. Kansas Sci. Bull. 53 (11): 689-690, figs 3 a-f. Holótipo macho, alótipo no SEMK; parátipos no DZUP, MBR e outras coleções conforme Michener, 1989: 690 .

Taxonomia. Michener, 1989: 635-638, 689 (considera representativo do grupo L. arnauellus e L. brunerii, como Perditomorpha s.str. e compara com diversas espécies).

leaena (Vachal). BrasiL, Minas Gerais, $1.000 \mathrm{~m}$ (localidade tipo).

Pasiphae leaena Vachal, 1909. Rev. d'Ent., Caen, 28: 38. Holótipo fêmea no MNHP.

Perditomorpha laena [sic]; Cure et al., 1993. Revista Ceres 40 (228): 138, 150.

Leioproctus leana [sic]; Michener, 1965. Bull. Amer. Mus. Nat. Hist. 130: 42.

Taxonomia. Moure, 1951. Dusenia, Curitiba, 2 (3): 191 (considera P. leaena sinônimo de $P$. brunerii).

Biologia. Cure et al., 1993: 138, 150 (em Sida sp., Malvaceae).

\section{Porterapis Michener, stat. nov.}

Lonchopria (Porterapis) Michener, 1989. Univ. Kansas Sci. Bull. 53 (11): 678-80. - Michener, 1997.

Sci. Papers, Nat. Hist. Mus., Univ. Kansas 1: 48.

Espécie-tipo: Lonchopria porteri Ruiz. Designação original.

porteri (Ruiz) comb.n. CHILE, Coquimbo, Valle del Choapa (localidade tipo).

Lonchopria porteri Ruiz, 1936. Rev. Chilena Hist. Nat. 40: 166-7. Síntipos: cinco fêmeas e dez machos no MHPN. 
Lonchopria (Porterapis) porteri; Michener, 1989. Univ. Kansas Sci. Bull. 53 (11): 678, 679, 680.

Taxonomia. Ruiz, 1944. Rev. Chilena Hist. Nat. 47: 228-9 (redescrição). fevereiro).

Biologia. Ruiz, 1936: 167 (em flores de Phrygilanthus tetrandrus, em

\section{Protodiscelis Brèthes.}

Protodiscelis Brèthes, 1909. An. Mus. Nac., Buenos Aires, 19: 245. - Melo, 1996. Revta bras. Ent. 40 (1): 97. - Michener, 1997. Sci. Papers, Nat. Hist. Mus., Univ. Kansas 1: 49.

Leioproctus (Protodiscelis); Michener, 1964. Systematic Zool. 13 (4): 187. - Michener, 1965. Bull. Amer. Mus. Nat. Hist. 130: 42. - Michener, 1989. Univ. Kansas Sci. Bull. 53 (11): 653.

Espécie-tipo: Protodiscelis fiebrigi Brèthes. Monotipia.

Taxonomia. Michener, 1965; 42 (considerou Protodiscelis como sub-gênero de Leioproctus). - Melo, 1996: 97 (tratou Protodiscelis como gênero).

echinodori Melo. Brasil, Minas Gerais, Viçosa (localidade tipo).

Protodiscelis echinodori Melo, 1996. Revta bras. Ent. 40 (1): 98-100, figs $3,4,7,9,10,12,15$. Holótipo fêmea, sete parátipos fêmeas e dois machos no DZUP; cinco parátipos fêmeas e um macho no MZSP; cinco parátipos fêmeas e um macho no MEUV; cinco parátipos fêmeas e um macho no SEMK.

Biologia. Melo, 1996: 97, 99 (coletados em flores de uma Alismataceae, Echinodorus grandiflorus, uma fêmea em flor de Rubiaceae, provavelmente Borreria. Fêmeas sem pólen nas escopas).

fiebrigi Brèthes. PARAGUAI, S. Bernardino (localidade tipo).

Protodiscelis fiebrigi Brèthes, 1909. An. Mus. Nac., Buenos Aires, 19: 246.

Síntipo macho no MBR.

Oediscelis fiebrigi; Ducke, 1912. Zool. Jahrb., Abt. Syst. 34: 83.

Leioproctus (Protodiscelis) fiebrigi; Michener, 1964. Systematic Zool. 13

(4): 187. - Michener, 1965. Bull. Amer. Mus. Nat. Hist. 130: 42.

Leioproctus (Protodiscelis) fiebrigi; Michener, 1989. Univ. Kansas Sci.

Bull. 53 (11): 653; figs 12 a-d.

Protodiscelis fiebreigi [sic]; Michener, 1989. Univ. Kansas Sci. Bull. 53 (11): 695 .

Taxonomia. Brèthes, 1909: 246 (descreveu fêmea e macho). - Michener, 1989: 695 (comenta problemas na descrição original e no material tipo desta espécie).

labrata Melo. BRASL, Minas Gerais: Viçosa (localidade tipo).

Protodiscelis labrata Melo, 1996. Revta bras. Ent., 40 (1): 97-98, figs 1, 2, 5, 8, 11, 13, 14. Holótipo macho no DZUP.

Biologia. Melo, 1996: 97 (em flores de uma Rubiaceae herbácea, provavelmente Borreria). 
palpalis (Ducke) comb.n. BRASIL, Ceará: Quixadá (localidade tipo).

Panurginus palpalis Ducke, 1908. Rev. d'Ent., Caen, 27: 67. Holótipo macho no MNHU.

Leioproctus (Protodiscelis) palpalis; Michener, 1989. Univ. Kansas Sci. Bull. 53 (11): 653.

Taxonomia. Michener, 1989: 653 (comentou que esta espécie provavelmente deveria ser incluida em Leioproctus (Protodiscelis).

Biologia. Ducke, 1910. Rev. Trim. Ceará, 24: 45 (visita flores de Alisma). spathigera (Michener) comb.n. BRASIL, São Paulo: Rio Claro (localidade tipo)

Leioproctus (Protodiscelis) spathigerus Michener, 1989. Univ. Kansas Sci. Bull. 53 (11): 695-696. Holótipo e parátipos fêmeas no SEMK; parátipos no DZUP.

Taxonomia. Melo, 1996. Revta bras. Ent. 40 (1): 97 - 100 (nota comparativa).

\section{Pygopasiphae Michener, stat. nov.}

Leioproctus (Pygopasiphae) Michener, 1989. Univ. Kansas Sci. Bull. 53 (11): 647. - Michener, 1997. Sci. Papers, Nat. Hist. Mus., Univ. Kansas 1: 51.

Espécie-tipo: Leioproctus (Pygopasiphae) mourellus Michener. Designação original.

mourella (Michener) comb.n. ARGENTINA, Catamarca, Andalgalá (localidade tipo); Buenos Aires.

Leioproctus (Pygopasiphae) mourellus Michener, 1989. Univ. Kansas Sci.

Bull. 53 (11): 693-694, figs 8 a-f, 10 h. Holótipo macho e alótipo fêmea, dois parátipos machos e uma fêmea no SEMK; dois parátipos machos no MBR; parátipos machos e fêmeas em outras coleções conforme Michener, 1989: 694.

Biologia. Michener, 1989: 694 (material tipo em flores de Prosopis chilensis, exceto um macho em Prosopis flexuosa; dois exemplares estilopizados, coletados com os parátipos; uma fêmea coletada em Prosopis alba).

wagneri (Vachal) comb.n. ARGENTINA, Santiago del Estero: bordo do rio Salado (localidade tipo); Gran Chaco, sul do rio Tapenaga; Mendoza, Chacras de Coria.

Pasiphae wagneri Vachal, 1909. Rev. d'Ent., Caen, 28; 39. Lectótipo fêmea e lectoalótipo no MNHP, selecionados por J. S. Moure em 1958, aqui designados.

Pasiphae armata Joergensen, 1912. Zool. Jahrb., Abt. Syst. 32: 103. Síntipos fêmeas no IML.

Leioproctus (Pygopasiphae) wagneri; Michener, 1989. Univ. Kansas Sci. Bull. 53 (11): 649.

Taxonomia. Ducke, 1912. Zool. Jahrb., Abt. Syst. 34: 77 (distribuição geográfica).

Biologia. Joergensen, 1912. An. Mus. Nac., Buenos Aires, 22: 302 ( $P$. armata em flores de Clematis hilarii, Prosopis alpataco e P. campestris). 


\section{Reedapis Michener, stat. nov.}

Leioproctus (Reedapis) Michener, 1989. Univ. Kansas Sci. Bull. 53 (11): 656.

Espécie-tipo: Leioproctus bathycyaneus Toro. Designação original.

Taxonomia. Toro, 1973. Rev. Chilena Ent. 7: 145-146 (chave, morfometria).

bathycyanea (Toro) comb.n. CHILE, Atacama: Travesía (localidade tipo), Canto del Agua, La Junta; Coquimbo, Samo-Alto, Ovalle, rio Los Moyes, San Marcos; Aconcágua, La Ligua.

Leioproctus bathycyaneus Toro, 1973. Rev. Chilena Ent. 7: 145-146, 166-169; figs 143-152.

Holótipo fêmea, alótipo e parátipos na CTV; parátipos machos e fêmeas na UCVC, AMNH, SEMK.

Leioproctus bathycyaneaus [sic] Toro, 1973. Rev. Chilena Ent. 7: 166.

Leioproctus (Reedapis) bathycyaneus; Michener, 1989. Univ. Kansas Sci. Bull. 53 (11): 657.

Taxonomia. Michener, 1989: 657; figs 14 h-i, 16 c; 19 g (nota descritiva).

melanocyanea (Toro) comb.n. CHILE, Antofagasta: norte de Paposo (localidade tipo).

Leioproctus melanocyaneus Toro, 1973. Rev. Chilena Ent. 7: 146, 165-6, figs 110-114. Holótipo fềmea e um parátipo fêmea na CTV

Leioproctus (Reedapis) melanocyaneus; Michener, 1989. Univ. Kansas Sci. Bull. 53 (11): 657.

semicyanea (Spinola) comb.n. CHILE, Províncias centrais, Atacama: Qda. Algodones, Frerina; Coquimbo: Huanguali, Plomos, San Marcos, Fray Jorge, Ovalle, Caimanes; Aconcágua: Zapallar, Rio Blanco; Valparaíso: Maitencillo, Horcones, Quilpué, Concón; Santiago: La Florida, San Fernando (Las Peñas); Concepción: Rancágua.

Colletes semi-cyanea Spinola, 1851, in Gay. Hist. Fis. Pol. Chile, Zool. 6: 221-222. Fêmea e macho no MIZT, a fêmea aqui designada como lectótipo e o macho como paralectótipo.

Biglossa caerulescens Friese, 1906. Ztschr. Hym. Dipt. 6: 378-9, 375 (chave). Síntipos, uma fêmea e dois machos. Um síntipo macho no MNHU.

Leioproctus (Reedapis) semicyaneus; Michener, 1989. Univ. Kansas Sci.

Bull. 53 (11): 657.

Taxonomia. Schrottky, 1907. An. Ci. Paraguayos 1 (7): 6-7 (redescrição). -Friese, 1908. Flora og Fauna 10: 17 (redescreveu como B. caerulescens, e chave). - Vachal, 1909. Rev. d'Ent., Caen, 28: 54 (como Lonchopria semicyanea). - Ducke, 1912. Zool. Jahrb., Abt. Syst. 34: 80 (L. semicyanea $=$ B. caerulescens). - Friese, 1912. Deutsch. Ent. Ztschr. : 366 (chave). - Ruiz, 1944. Rev. Chilena Hist. Nat. 47: 224-5 (redescrição). - Toro, 1973. Rev. Chilena Ent. 7: 162-165; figs 133-142 (redescrição, distribuição geográfica). 


\section{Sarocolletes Michener}

Leioproctus (Sarocolletes) Michener, 1989. Univ. Kansas Sci. Bull. 53 (11): 643. - Michener, 1997. Sci. Papers, Nat. Hist. Mus., Univ. Kansas 1: 53.

Sarocolletes; Moure \& Urban, [1995] 1992. Acta Biol. Par., Curitiba, 21 (1-4): 113.

Espécie-tipo: Lonchopria rufipennis Cockerell. Designação original.

duplex (Michener). ARgEnTINA, Santa Fé, Departamento Garay, Colonia Masoias (localidade tipo)

Leioproctus (Sarocolletes) duplex Michener, 1989. Univ. Kansas Sci. Bull. 53 (11): 692-3, figs 7 a-h. Holótipo macho, alótipo fêmea e sete parátipos machos no SEMK; dois parátipos machos no MBR.

Sarocolletes duplex; Moure \& Urban, [1995] 1992. Acta Biol. Par., Curitiba, 21 (1-4): 118.

Taxonomia. Moure \& Urban, [1995] 1992: 118-119 (nota comparativa).

fulva Moure \& Urban. BRASIL, Bahia, Casa Nova, próximo de Sobradinho (localidade tipo). Holótipo macho, alótipo fêmea e parátipos machos e fêmeas no DZUP.

Sarocolletes fulva Moure \& Urban, [1995] 1992. Acta Biol. Par., Curitiba, 21 (1-4): 114- 115, figs 5-9.

Biologia. Moure \& Urban, [1995] 1992: 115 (em flores de Malvaceae).

guaritarum Urban. BRASIL, Rio Grande do Sul: Caçapava do Sul (localidade tipo) Sarocolletes guaritarum Urban, 1995. Revta bras. Zool. 12 (2): 401-2. Holótipo fêmea e parátipos fêmeas no DZUP; parátipos fêmeas no UCRS.

minor Moure \& Urban. ARgENTINA, Formosa, Laguna Blanca (localidade tipo).

Sarocolletes minor Moure \& Urban, [1995] 1992. Acta Biol. Par., Curitiba, 21 (1-4): 115-116, fig. 2. Holótipo macho, alótipo fêmea e parátipo macho no DZUP.

pallida Moure \& Urban, ARgENTINA, Tacanas (localidade tipo).

Sarocolletes pallida Moure \& Urban, [1995] 1992. Acta Biol. Par., Curitiba, 21 (1-4): 116-117, fig. 3. Holótipo macho e alótipo fêmea no DZUP.

rufipennis (Cockerell) ARgenTINA, Córdoba, Catamarca: Carcarañá (localidade tipo).

Lonchopria rufipennis Cockerell, 1917. Ann. Mag. Nat. Hist. (8) 20: 439. Síntipo macho no USNM.

Lonchopria rufipennis parva Cockerell, 1917. Ann. Mag. Nat. Hist. (8) 20: 440. Síntipo macho no USNM.

Sarocolletes rufipennis; Moure \& Urban, [1995] 1992. Acta Biol. Par., Curitiba, 21 (1-4): 117.

Taxonomia. Cockerell, 1917: 439 (descreveu macho e fêmea). - Michener, 1989: 646, fig. $10 \mathrm{~g}$ (L. (S.) rufipennis parva provável sinônimo de L. rufipennis). 
- Moure \& Urban, [1995] 1992: 117, fig. 1 (redescrevem e consideram a variedade L. rufipennis parva Cockerell na sinonímia).

\section{Spinolapis Moure.}

Pasiphae Spinola, 1851, in Gay. Hist. Fis. Pol. Chile, Zool., 6: 226 (nom. praeoc. Latreille, 1819, N. Dict., 30: 73, Crust.).

Espécie-tipo: Pasiphae caerulescens Spinola. Designação de Sandhouse, 1943: 585.

Brachyglossula; Sandhouse, 1943. Proc. U. S. Natl. Mus. 92: 585.

Spinolapis Moure, 1951. Dusenia, Curitiba, 2 (3): 193-194 (nom. n. para Pasiphae Spinola, 1851). Michener, 1997. Sci. Papers, Nat. Hist. Mus., Univ. Kansas 1: 55.

Leioproctus (Spinolapis); Michener, 1964. Systematic Zool. 13 (4): 187. - Michener, 1965. Bull. Amer. Mus. Nat. Hist. 130: 41. - Michener, 1989. Univ. Kansas Sci. Bull. 53 (11): 658.

Taxonomia. Sandhouse, 1943: 585 (considerou Pasiphae Spinola, non Latreille, igual a Brachyglossula Hedicke, e designou B. caerulescens como espécie-tipo). - Michener, 1989: 658-9; figs $15 \mathrm{a}-\mathrm{j}, 16 \mathrm{~g}$ (redescrição, nota comparativa).

caerulescens (Spinola). CHILE, Santa Rosa (localidade tipo).

Pasiphae caerulescens Spinola, 1851, in Gay. Hist. Fis. Pol. Chile, Zool., 6: 228. Fêmea e macho no MIZT, o macho aqui designado lectótipo e a fêmea paralectótipo.

Brachyglossula caerulescens; Sandhouse, 1943. Proc. U. S. Natl. Mus. 92: 585.

Spinolapis caerulescens; Moure, 1951. Dusenia, Curitiba, 2 (3): 193.

Leioproctus (Spinolapis) caerulescens; Michener, 1964. Systematic Zool.

13 (4): 187. - Michener, 1989. Univ. Kansas Sci. Bull. 53 (11): 659.

Taxonomia. - Moure, 1951: 193 (nota comparativa). - Michener, 1989: 659, fig. $15 \mathrm{a}-\mathrm{j}, 660$, fig. $16 \mathrm{~g}$.

cyanea (Cockerell) comb.n. ARGENTINA, Tierra del Fuego, Rio McClelland (localidade tipo). CHILE, Magallanes.

Pasiphae cyanea Cockerell, 1915. Ann. Mag. Nat. Hist. (8) 15: 341-342. Síntipo fêmea no BMNH.

Leioproctus (Bicolletes) cerdai Toro, 1973. An. Mus. Hist. Nat., Valparaíso, 6: 207-210, figs 136-139. CHILE, Magallanes. Holótipo fêmea, na CTV.

Leioproctus (Spinolapis) cyaneus; Michener, 1989. Univ. Kansas Sci. Bull. 53 (11): 659.

Taxonomia. Michener, 1989: 659 (colocou L. cerdai Toro, 1973 na sinonímia desta espécie).

melanura (Cockerell) comb.n. ARGENTINA, Chubut (localidade tipo).

Pasiphae cyanea melanura Cockerell, 1917. Ann. Mag. Nat. Hist. (8) 20: 236. Tipo fêmea no USNM.

Leioproctus (Spinolapis) melanurus; Michener, 1989. Univ. Kansas Sci.

Bull. 53 (11): 659. 
Taxonomia. Michener, 1964. Systematic Zool. 13 (4): 187 (reconheceu como espécie). - Michener, 1989: 659 (colocou L. (S.) melanurus, com dúvidas, entre as espécies de Leioproctus (Spinolapis).

\section{Tetraglossula Ogloblin}

Tetraglossula Ogloblin, 1948. Notas Mus. La Plata, Zool., 13 (106): 165. - Michener, 1964. Systematic Zool. 13 (4): 189. - Michener, 1965. Bull. Amer. Mus. Nat. Hist. 130: 40. - Melo, 1996. Revta bras. Ent. 40 (1): 97. - Michener, 1997. Sci. Papers, Nat. Hist. Mus., Univ. Kansas 1: 57.

Espécie-tipo: Tetraglossula deltivaga Ogloblin. Designação original.

Leioproctus (Tetraglossula); Michener, 1989. Univ. Kansas Sci. Bull. 53 (11): 654.

Taxonomia. Michener, 1989: 654-656 (redescrição como subgênero de Leioproctus). - Melo, 1996. Revta. bras. Ent. 40 (1): 97 (considera como gênero). anthracina (Michener) comb.n. BRASIL, São Paulo: São Carlos (localidade tipo); Paraná, Curitiba.

Leioproctus (Tetraglossula) anthracinus Michener, 1989. Univ. Kansas

Sci. Bull. 53 (11): 696. Holótipo macho e alótipo fêmea, sete parátipos machos e cinco parátipos fêmeas no SEMK; dois parátipos no DZUP.

bigamica (Strand). PARAGUAI, Asunción (localidade tipo); Sapucay. ARGENTINA, Misiones, Pindapoy.

Bicolletes bigamica Strand, 1910. Zool. Jahrb., Abt. Syst. 29: 463. Síntipos macho e fêmea no MNHU.

Bicolletes stigmaticus Strand, 1910. Zool. Jahrb., Abt. Syst. 29: 464. Nome proposto para a fềmea descrita como bigamica, caso fosse de outra espécie.

Tetraglossula birabeni Ogloblin, 1948. Notas Mus. La Plata 13: 169-172; figs 8-15. Holótipo macho no MLP.

Tetraglossula bigamica; Moure, 1951. Dusenia, Curitiba, 2 (3): 191. Michener, 1964. Systematic Zool. 13 (4): 189.

Leioproctus (Tetraglossula) bigamicus; Michener, 1989. Univ. Kansas Sci. Bull. 53 (11): 656.

Taxonomia. Moure, 1951: 191 (colocou T. birabeni na sinonímia).

Morfologia. Gimenes, 1991. Revta bras. Ent. 35 (2): 414-416, 420; figs 1 a-c, 3 b, 4 14-17 (escopa esternal e coleta de pólem em Ludwigia elegans, Onagraceae).

Biologia. Schlindwein, 1995. Wildbienen und ihre Trachtpflanzen..., Stuttgart: 89 (Ludwigia mayor, L. peploides, Onagraceae).

deltivaga Ogloblin. ARgENTINA, Buenos Aires (localidade tipo): Dique Luján.

Tetraglossula deltivaga, Ogloblin, 1948. Notas Mus. La Plata, Zool. 13:

166-9; figs 1-7. Holótipo macho, alótipo e parátipos no MLP.

Leioproctus (Tetraglossula) deltivagus; Michener, 1989. Univ. Kansas Sci.

Bull. 53 (11): 656. 
fucosa (Michener) comb.n. Brasil, Pará: Cachimbo; São Paulo: São Carlos (localidade tipo).

Leioproctus (Tetraglossula) fucosus Michener, 1989. Univ. Kansas Sci. Bull. 53 (11): 656, 697; figs 13 a-k, 16 f. Holótipo macho, alótipo e parátipos no SEMK; parátipos no DZUP, USNM e AMNH.

\section{Torocolletes Michener, stat. nov.}

Leioproctus (Torocolletes) Michener, 1989. Univ. Kansas Sci. Bull., 53 (11): 651, figs 16a; 115-132. - Michener, 1997. Sci. Papers, Nat. Hist. Mus., Univ. Kansas 1: 58.

Espécie-tipo: Lonchopria fazii Herbst. Designação original.

Taxonomia. Michener, 1965. Bull. Amer. Mus. Nat. Hist. 130: 41 (considerou as duas espécies deste gênero: fazii e ibanezii na listagem de Leioproctus, sub-gênero Lonchopria).

fazii (Herbst) comb.n. CHILE, Valparaiso (localidade tipo): El Salto, Quilpué, Villa Alemana; Aconcagua; Santiago.

Lonchopria fazii Herbst, 1923. Rev. Chilena Hist. Nat. 27: 74-75. Síntipos no MNHU.

Leioproctus (Lonchopria) fazii; Michener, 1965. Bull. Amer. Mus. Nat. Hist. 130: 41.

Leioproctus fazii; Toro, 1973. Rev. Chilena Ent. 7: 146.

Leioproctus (Torocolletes) fazii; Michener, 1989. Univ. Kansas Sci. Bull. 53 (11): 651.

Taxonomia. Toro, 1973: 146 (chave), 157-159 (redescrição); figs 115-123.

Biologia. Herbst, 1923: 75 (os machos voam no princípio do mês de agosto, são mais numerosos no início de setembro; as fêmeas aparecem nos meados de setembro e o período de vôo termina em meados de outubro. Ambos os sexos nas flores de Azara celastrina, Adesmia arborea, Schinus latifolius). - Ruiz, 1944. Rev. Chilena Hist. Nat. 47: 225 (repetição dos dados de Herbst, 1923).

ibanezii (Ruiz) comb.n. CHILE, Valparaiso (localidade tipo), Curauma, Cuesta La Dormida.

Lonchopria ibanezii Ruiz, 1944. Rev. Chilena Hist. Nat. 47: 226. Holótipo fềmea, alótipo macho e várias fêmeas e machos no MHPN.

Leioproctus (Lonchopria) ibanezii; Michener, 1965. Bull. Amer. Mus. Nat. Hist. 130: 41.

Leioproctus ibanezii; Toro, 1973. Rev. Chilena Ent. 7: 146.

Leioproctus (Torocolletes) ibanezii; Michener, 1989. Univ. Kansas Sci. Bull. 53 (11): 651.

Taxonomia. Toro, 1973: 146 (chave), 159-162 (redescrição); figs 124-132; 171-172 dados morfométricos.

Biologia. Ruiz, 1944: 226 (sobre flores de Umbelifera em dezembro). 


\section{REFERÊNCIAS BIBLIOGRÁFICAS}

Melo, G.A.R. 1996. Two new Brazilian Bees of the genus Protodiscelis (Hymenoptera, Colletidae). Revta bras. Ent. 40 (1): 97-100.

MiCHENER, C.D. 1944. Comparative external morphology, phylogeny, and a classification of the bees. Bull. Amer. Mus. Nat. Hist. 82: 151-326.

. 1964. The Possible Use of Uninominal Nomenclature to Increase the Stability of Names in Biology. Systematic Zool. 13 (4): 182-190. 1965. A Classification of the Bees of the Australian and South Pacific Regions. Bull. Amer. Mus. Nat. Hist. 130: 1-362.

1989. Classification of the American Colletinae (Hymenoptera, Apoidea).

Univ. Kansas Sci. Bull. 53 (11): 623-703.

1997. Genus-Group Names of Bees and Supplemental Family-Group

Names. Sci. Papers, Nat. Hist. Mus., Univ. Kansas 1: 1-81.

MOURE, J.S. 1945. Contribuição para o conhecimento dos Diphaglossinae, particularmente Ptiloglossa. Arq. Mus. Paranaense, Curitiba, 4: 137-178.

1948. Notas sobre algumas abelhas de Tacanas, Tucumán, Argentina. (Hymenopt. Apoidea). Rev. Ent., Rio de Janeiro, 19 (1/2): 313-346.

1949. Notas sobre algunas abejas de Tacanas, Tucumán, Argentina. II.

(Hymenopt. Apoidea). Rev. Ent., Rio de Janeiro, 20 (1/3): 437-460.

1951. Notas sobre abelhas do antigo gênero Pasiphae (Hymenopt. Apoidea). Dusenia, Curitiba, 2 (3): 189-198.

1954. Novas notas sôbre abelhas do antigo gênero Pasiphae (Hymenopt.

- Apoidea). Dusenia, Curitiba, 5 (3/4): 165-190.

Moure, J.S. \& P.D. HURD JR. 1987. An Annotated Catalog of the Halictid Bees

of the Western Hemisphere. Washington, Smithsonian Institution Press, VII $+405 \mathrm{p}$.

Moure, J.S. \& D. URBAN. 1992. Sarocolletes Michener, 1989, status nov. e espécies novas (Hymenoptera, Apoidea, Colletidae). Acta Biol. Par., Curitiba, 21 (1-4): 113-120 [1995].

NAscimento, P.T.R. 1979. Catálogo de tipos Entomológicos do Museu Goeldi. Hymenoptera. Bol. Mus. Paraense Emílio Goeldi, n. s. Zoologia, 98: 1-18.

UrBAN, D. 1995. Espécies novas de Paracolletini e Panurginae do sul do Brasil e Argentina (Hymenoptera, Apoidea). Revta bras. Zool. 12 (2): 397-405.

Recebido em 19.XI.1998; aceito em 23.III.1999. 


\section{ÍNDICE DOS TAXA DE PARACOLLETINI}

abdominalis (Joergensen), Bicolletes abdominis Michener $=$ abdominalis . Actenosigynes Moure; Graf \& Urban Aeganopria Moure. aenea (Friese), Biglossidia alismatis (Ducke), Bicolletes. alopex (Cockerell), Biglossidia andina (Herbst), Bicolletes annectens (Michener), Lonchoprella anthracina (Michener), Tetraglossula armata Friese = chalybaea armata Joergensen $=$ wagneri arnauellus Michener $=$ arnaui, $P$. arnaui Moure, Nomiocolletes. arnaui Moure, Perditomorpha. atacama (Toro \& Rojas), Bicolletes... basirufa (Schrottky), Bicolletes. bathycyaneaus $[\mathbf{s i c}]=$ bathycyanea . bathycyanea (Toro), Reedapis. Belopria Moure. bernardinensis Strand = psaenythioides .. 23 bicellularis (Ducke), Bicolletes. Bicolletes Friese. bigamica (Strand), Tetraglossula . Biglossa Friese.

Biglossidia Moure

birabeni Ogloblin = bigamica boliviensis Vachal = bouvieri . bouvieri (Vachal), Brachyglossula. Brachyglossula Hedicke brunerii Ashmead, Perditomorpha caerulescens Friese $=$ semicyanea . caerulescens (Spinola), Spinolapis cearensis (Ducke), Nomiocolletes. Cephalocolletes Michener cerdai Toro $=$ cyanea . cestri Ducke $=$ iheringi chalybaea (Friese), Biglossidia chalybea $=$ chalybaea. Chilicolletes, Michener chrysostoma (Cockerell), Bicolletes.... cingulata (Moure), Ctenosibyne. colaratipes $[\mathbf{s i c}]=$ coloratipes coloratipes (Cockerell), Bicolletes crassipunctata Urban, Cephalocolletes.....19 cristariae Joergensen, Holmbergeria Ctenosibyne Moure cyanea (Cockerell), Spinolapis. deceptrix Moure, Biglossidia...... decolorata (Ducke), Bicolletes .................... 8

delahozii (Toro), Chilicolletes .................... 20

deltivaga Ogloblin, Tetraglossula ............ 39

Drachyglossula $[\mathbf{s i c}]=$ Brachyglossula $\ldots . . .6$

duplex (Michener), Sarocolletes ................ 37

echinodori Melo, Protodiscelis ................. 34

ecuadoria (Friese), Lonchorhyncha ......... 29

Edwyniana Moure........................................ 21

erithrogaster (Toro \& Rojas), Bicolletes... 9

Ethalonchopria Michener.......................... 22

Eulonchopria Brèthes ................................. 23

eulonchopriodes (Michener), Bicolletes.... 9

fasciata (Schrottky), Bicolletes................... 9

fazii (Herbst), Torocolletes ........................ 40

ferruginea Moure, Bicolletes ...................... 9

fiebreigi $[$ sic $]=$ fiebrigi .............................. 34

fiebrigi Brèthes, Protodiscelis ................... 34

flavescens (Friese), Eulonchopria ............ 23

flavescens Friese = psaenythioides ........... 24

flavicornis (Spinola), Edwyniana .............. 21

flavitarsus (Toro), Bicolletes ....................... 9

franki Friese, Bicolletes ............................. 9

friesei (Joergensen), Bicolletes .................. 10

fucosa (Michener), Tetraglossula ............. 40

fulva Moure \& Urban, Sarocolletes.......... 37

fulvoniger Michener, Actenosigynes........... 4 gaullei (Vachal), Ethalonchopria ............. 22 Glossopasiphe Michener ............................ 24

guaritarum Urban, Sarocolletes ............... 37

Halictanthrena Ducke ................................ 24

herbsti Vachal = zonalis ........................... 29

herrerae (Toro), Edwyniana ...................... 21

Hexantheda Ogloblin................................. 25

Holmbergeria Joergensen ........................... 25

Holmbergia $[$ sic] $=$ Holmbergeria ............ 25

Hoplocolletes Michener............................ 26

ibanezii (Ruiz), Torocolletes ..................... 40

iheringi (Schrottky), Bicolletes.................. 10

inca (Cockerell), Biglossidia .................... 17

inconspicua (Michener), Perditomorpha. 33

isabelae Urban, Cephalocolletes .............. 19

jenseni (Friese), Nomiocolletes.................. 31

joergenseni (Friese), Nomiocolletes ......... 31

Kylopasiphae Michener ............................ 27

labrata Melo, Protodiscelis ....................... 34

laena $[\mathbf{s i c}]=$ leaena .................................. 33

laticeps (Friese), Cephalocolletes............. 19

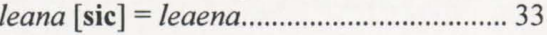


leaena (Vachal), Perditomorpha

Leoiproctus $[\mathbf{s i c}]=$ Leioproctus .

leucostoma (Cockerell), Bicolletes

limbella (Vachal), Ethalonchopria

Lonchoprella Michener

Lonchopria Vachal

Lonchorhyncha Michener

longicornis (Michener), Biglossidia

longipes (Joergensen), Bicolletes.

lucidula (Cockerell), Bicolletes

luteipes (Friese), Lonchopria . malpighiacearum (Ducke), Halictanthrena

25

marginata Spinola $=$ zonalis

melanocyanea (Toro), Reedapis....

melanura (Cockerell), Spinolapis

minor Moure \& Urban, Sarocolletes

missionica Ogloblin, Hexantheda.

moerens (Vachal), Bicolletes

mourei (Toro), Edwyniana ....

mourella (Michener), Pygopasiphae...

neotropica Friese, Bicolletes.

nigriceps Friese, Bicolletes

Niltonia Moure.

nitidior Moure, Belopria.

nivosa (Vachal), Aeganopria

Nomiocolletes Brèthes

oaxacana (Michener), Eulonchopria.....

orientalis (Vachal), Bicolletes ....

pallida Moure \& Urban, Sarocolletes

palpalis (Ducke), Protodiscelis

pampeana Urban, Bicolletes.

Pasiphae (ver Spinolapis)

$11,12,13,14,18,21,22,33,35,38,41$

paraguayensis (Schrottky), Bicolletes...

parva Cockerell = rufipennis

penai (Toro \& Rojas), Bicolletes.

penal $[$ sic $]=$ penai

Perditomorpha Ashmead.

perezi (Toro \& Rojas), Bicolletes

perornata Cockerell $=$ bouvieri .

personata $[\mathbf{s i c}]=$ perornata

peruviana (Cockerell), Bicolletes.....

pharcidodes Moure, Bicolletes

plaumanni (Michener), Glossopasiphae .. 24

Porterapis Michener.

porteri (Ruiz), Porterapis .

Protodiscelis Brèthes

pruinosa (Michener), Kylopasiphae

psaenythioides Brèthes, Eulonchopria
33 pseudozonata Moure, Bicolletes 13

9 punctatissima Michener, Eulonchopria .... 24

10 Pygopasiphae Michener............................. 35

23 Reedapis Michener...................................... 36

27 robertsi (Michener), Biglossidia ................ 18

27 ruber $=$ rubra ............................................. 13

29 rubra (Toro \& Rojas), Bicolletes ............... 13

17 rubriventris (Friese), Holmbergeria ......... 26

11 rufipennis (Cockerell), Sarocolletes .......... 37

11 rufithorax $[$ sic] $=$ rufitorax ....................... 28

27 rufitorax Ruiz, Lonchopria ........................ 28

rufiventris (Spinola), Bicolletes ................ 14

rufocaerulea Friese = bouvieri .................. 18

rufocoerulea $[$ sic $]=$ rufocaerulea $\ldots \ldots \ldots \ldots . . . .18$

rugata Urban, Cephalocolletes ................. 19

saltensis Friese, Bicolletes ........................ 14

37 Sarocolletes Michener..................................37

25 semicyanea (Spinola), Reedapis ................ 36

11 seydi Strand, Bicolletes ............................... 14

21 similis (Friese), Lonchopria ........................ 28

35 simplicicrus (Michener), Nomiocolletes .. 32

11 spathigera (Michener), Protodiscelis ....... 35

12 spegazzini (Joergensen), Bicolletes............ 14

30 spegazzinii $[$ sic] = spegazzini ..................... 14

5 spinolae Dalla Torre $=$ zonalis ................... 28

5 Spinolapis Moure ......................................... 38

30 sticta Moure, Bicolletes.............................. 14

23 stigmaticus Strand = bigamica ................... 39

12 stillborhina Moure, Bicolletes................... 15

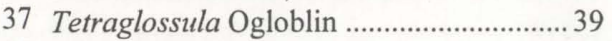

35 thoracica Friese, Biglossa ......................... 15

12 tomentifera Strand = psaenythioides ......... 23

Torocolletes Michener .................................. 40

tristis (Spinola), Edwyniana........................22

ventralis (Friese), Hoplocolletes ............... 26

virgilii Moure, Niltonia ............................... 30

wagenknechti (Toro \& Rojas), Bicolletes 15

wagneri (Vachal), Pygopasiphae .............. 35

zonalis (Reed), Lonchopria ........................ 28

zonata Moure, Belopria ................................ 6

\section{ÍNDICE DAS PLANTAS}

absinthioides, Tessaria.

Abutilon $12,32,33$

Acacia. $11,16,23,24,31,32$

Achyrophorus

Adesmia

africana, Tamarix.

alba, Prosopis . 
albicaulis, Senecio

11 Cynara .11

Alisma

7, 35 denudatum, Gymnocalycium

Alismataceae

34 divaricata, Larrea ... 7, 11, 16, 18, 26, 27, 32

alpataco, Prosopis.......11, 16, 19, 31, 32, 35 Echinodorus

7,34

angustissima, Acacia

24 effusa, Baccharis

11,32

arborea, Adesmia

40 eichleri, Cajophora

.4

arechavaletae, Cajophora

$9,10,12$ elaeagnifolium, Solanum

argentinum, Lycium.

15 elegans, Ludwigia

arvensis, Convolvulus

12 Eryngium

22,31

Aster

11 Escallonia .22

astragalina, Glycyrrhiza

16 Euphorbiaceae.

.10

atriplicifolium, Solanum

16 Flaveria

10,11

augustis, Acacia.

24 flexuosa, Prosopis

35

Azara

40 fortunei, Paulownia.

.30

Baccharis 11, 15, 17, 24, 31, 32 Fuchsia.

Bidens

11, 15 furcata, Acacia

$11,16,31,32$

Bignoniaceae

30 genistoides, Cyclolepis

32

Blumenbachia

10, 12 glauca, Pascalia

11

bonariensis, Sphaeralcea

7, 10, 12, 14 glaucus, Achyrophorus

11

Borreria

8, 34 Glechon

10,12

brunneogemmia, Opuntia.

19, 20 Glycyrrhiza

16

Bulnesia

$16,18,27$

gnaphalii, $\mathrm{Cr}$

10

Cactaceae

19, 20 gracile, Lycium

. .15

Caesalpinia.

11 grandiflorus, Echinodorus.

.34

Cajophora.

4, 9, 10, 12 Grindelia

11,32

campestris, Prosopis ..11, 16, 19, 31, 32, 35

Grobowskya

.16

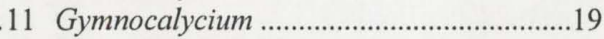

cardunculus, Cynara

40 Gymnophytum.

.7

Centaurea

11 hermanniae, Convolvulus.

12

Cereus

28 Heterothalamus.

$15,31,32$

10 hilarii, Clematis

$12,16,35$

15 horridum, Eryngium

..31

chilense, Lycium

.35 hualtata, Senecio..

.22

chilensis, Prosopis

11 Hypochoeris 31

Clematis $12,16,35$

Hyptis ...8

coerulescens, Baccharis marginalis...

collina, Sphaeralcea

Hysterionica.

11

12 hysterionoides, Parthenium .........................11

Compositae [Asteraceae]

11 hysterophorus, Parchenium

Composta

24 incisa, Cunila

contrahyerba, Flaveria.

Convolvulaceae

10, 11 Inga

.23

12 inodorum, Nothoscordum ...........................10

Convolvulus

12 insignis, Blumenbachia.

10,12

Cristaria

10, 14, 26 Jacaranda

30

Croton....

10,31

1 jasionoides, Hysterionica

cuneata, Monnina

cuneifolia, Larrea

Cunila

19 juncea, Baccharis

18, 27 Lamiaceae

10 lanceolatum, Circium

Curcubita.

30 Larrea

$11,16,18,26,27,32$

Cuscuta...

12 latifolia, Cristaria

26

Cyclolepis

32 latifolius, Schinus. 
Leguminosae

11 Phrygilanthus 34

leprosa, Sida

12 Physalis.....

15,19

leptolobus, Senecio

12 pingrea, Baccharis

leucantha, Bidens....

11, 15 pinnatus, Senecio.....

$11,15,32$

linearifolia, Solidago

11 piperitum, Phoeniculum

linifolius, Aster.

11 polycephalum, Gymnophytum.

7

litoralis, Cereus.

28 Polygalaceae

19,20

Loasa

14, 22, 29 precox, Caesalpinia

11

Loasaceae

10 Prosopis.

$11,16,18,19,27,31,32,35$

loasifolia, Cristaria

10, 14, 26 puberula, Jacaranda

30

Ludwigia

39 pulchella, Grindelia

11,32

Lycium

15 Quillaja.

29

macrostemma, Fuchsia .

22 racemosa, Cuscuta

12

Malpighiaceae

25 retama, Bulnesia $16,18,27$

Malva

12 rotundifolia, Malva 12

Malvaceae ....

12, 26, 33, 37 Rubiaceae

mammulosus, Notocactus ...

19, 20 rubra, Escallonia..

22

marginalis, Baccharis

17, 32 sagitallis, Baccharis

mayor, Ludwigia

39 salicifolia, Baccharis.

$11,15,31,32$

Medicago.

16 saponaria, Quillaja 29

melitensis, Centaurea.

11 sativa, Medicago ... 16

mendocina [sic], Abutilon.

32 scabriosoides, Telesperma

32

mendocina, Sphaeralcea .

12 Schinus

10,40

mendocinum, Abutilon

12 sellowii, Notocactus.

19,20

mendocinus, Senecio....

11 Senecio

$11,12,15,22,32$

microptera, Ximenedia

11, 31 serrulata, Baccharis.

11,15

Mimosoidea.

24 Sida.

12,33

molle, Schinus

10 Solanaceae.

.. 15

Monnina

19 Solanum

15,22

nigra, Prosopis.

18 Solidago.

Nothoscordum

10 spartioides, Heterothalamus ......... 15, 31, 32

Notocactus.

19, 20 speciosa, Grindelia

nudicaule, Eryngium.

31 Sphaeralcea.

$10,12,14$

obtusa, Grobowskya

16 stipellatus, Senecio.....

officinales, Taraxacum

11 strombulifera, Prosopis.

39 suaveolens, Hyptis.....

Opuntia

19, 20 subulata, Baccharis

$11,15,32$

19,20

ottonis, Notocactus.

succineus, Notocactus

19,20

Oxalis

12 sulphurea, Opuntia.

pampasica, Hypochoeris.

31 Tamarix 12

paniculatum, Eryngium.

22 Taraxacum.

papillosa, Triscis....

11 Telesperma

17 Tessaria

11,32

Parthenium.

parviflora, Malva

12 thermarum, Croton.

Pascalia.

11 thymoides, Glechon

10,31

pauciflorum, Abutilon...

33 Triscis

10,12

Paulownia

peploides, Ludwigia

39 verticillata, Borreria

Phoeniculum

32 viridirubra, Opuntia. 
viscosa, Physalis .................................15, 19

Ximenedia .......................................... 11, 31

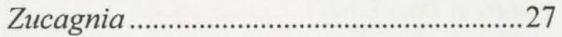

\section{ÍNDICE DOS PARASITAS}

Isepeolus luctuosus Spinola (em Lonchopria marginata) ............................................... 29

Isepeolus viperinus Friese (em Lonchopria marginata) ................................................. 29

Sphecodes friesei Herbst (em Lonchopria marginata) ........................................................ 29 